\title{
The B3-VLA quasar sample
}

\author{
M. Vigotti ${ }^{1}$, G. Vettolani ${ }^{1}$, R. Merighi ${ }^{2}$, J.F. Lahulla ${ }^{3}$, and M. Pedani ${ }^{1}$ \\ 1 Istituto di Radioastronomia del CNR, Bologna, Italy \\ 2 Osservatorio Astronomico di Bologna, Italy \\ 3 Observatorio Astronomico de Madrid (O.A.N.-I.G.N.) Spain
}

Received July 22; accepted September 23, 1996

\begin{abstract}
A new low frequency radio selected Sample of 125 Quasars complete down to $100 \mathrm{mJy}$ at $408 \mathrm{MHz}$ is presented in this paper. The sample is a part of the B3-VLA sample: 1050 radiosources selected from the B3 catalogue at $408 \mathrm{MHz}$ and observed at the VLA $(1465 \mathrm{MHz}, \mathrm{C}$ and A configurations). Out of the 352 sources, identified on the POSS-I down to $m_{\mathrm{r}} \sim 20.0,172$ are quasar candidates. In this paper we give the final assessment of the quasar sample from spectroscopic observations of the candidates. The final complete quasar sample consists of 125 objects. Furthermore $3 \mathrm{Bl}$ Lac objects have been identified and two Bl Lac candidates.
\end{abstract}

Key words: galaxies: active — quasars: general — radio continuum: general

\section{Introduction}

Quasars were first discovered through the optical identification of radio sources. Optical searches, based on different criteria from multicolor selection to grism searches, have produced a large number of optically selected quasar and have shown that radio loud quasars are really a fraction of the quasar population. However, optical multicolor searches have their own problems in completeness if, for instance, a large fraction of obscured quasars exists (Webster et al. 1995). In principle radio quasars samples do not suffer from a bias of this kind and, therefore, studying their color distribution, they should provide a direct test on the number of obscured quasars.

In the last years, a renewed interest is also raising in radio samples selected at low frequency because they provide an effective way to test the Unified Schemes (US) for

Send offprint requests to: M. Vigotti

* Tables 4, 5, 6 and Figs. 1, 2, 3, 4, 6 are also available in electronic form at the CDS via anonymous ftp to: cdsarc.ustrasbg.fr (130.79.128.5) or via http://cdsweb.u-strasbg.fr/ Abstract.html extragalactic radiosources (Barthel 1989) through, for instance, a direct comparison of the observed angular sizes of radiogalaxies and quasars in the same redshift range.

Quasar samples selected at meter-wavelength are preferable with respect to high frequency selected samples because the source selection is largely based on their lobe emission. On the contrary flat spectrum sources, predominant in high frequency selected samples, are mostly core-dominated cases where the relativistic beaming might introduce serious selection effects.

Although US are generally accepted, some authors found observational evidences which cannot be explained by this scheme and that require a more complex treatment of the AGN phenomenon. Using 3CR, MQS, 1JY samples and an earlier version of the present B3-VLA quasar sample (see Singal 1993 and references therein), Singal finds several discrepancies between the predictions of US and the observational data. For instance, the observed number of quasars versus total source number, namely the quasar fraction $\left(f_{\mathrm{q}}\right)$ changes with the limiting flux of the sample. In the MQS sample, Kapahi et al. (1996) show that apparent sizes of radiogalaxies and quasars have almost an equal distribution at any redshift in contradiction with the US prediction. A similar result has been found by Blundell et al. (1996), using 3CR, 5C, 6C and 8C samples.

In a future paper, using all the available parameters of radiogalaxies and quasars of the B3-VLA sample (apparent diameters, flux, power, redshifts), we will verify the predictions of the US.

In Sect. 2 we present the final QSO sample based on spectroscopic observations reported in Vigotti et al. (1990), Lahulla et al. (1991) and further observations of the remaining candidates described in Sect. 3 of the present paper. Section 4 contains comments on some individual sources, and finally Sect. 5 summarizes the present data. 


\section{The B3-VLA quasar sample}

\subsection{The radio sample}

The B3 radiosource catalogue (Ficarra et al. 1985), lists 13354 sources brighter than $100 \mathrm{mJy}$ at $408 \mathrm{MHz}$ and covers 0.78 steradians in a sky strip from $37^{\circ}$ to $47^{\circ}$, all hour angles.

From the B3, down to the catalogue limit of $0.1 \mathrm{Jy}$, Vigotti et al. (1989) selected five complete subsamples separated by equal increments in logarithmic flux density and containing approximately the same number of sources, through the choice of different declination limits for each subsample. Furthermore, the right ascention range was restricted to exclude sky areas at low galactic latitude (R.A. $23^{\mathrm{h}} 00^{\mathrm{m}}-03^{\mathrm{h}} 00^{\mathrm{m}}$ and $\left.07^{\mathrm{h}} 00^{\mathrm{m}}-15^{\mathrm{h}} 00^{\mathrm{m}}\right)$.

From VLA maps in C configuration at $1465 \mathrm{MHz}$ for the 1103 sources in the sample, Vigotti et al. (1989) obtained radio positions (accurate to 0.5 arcsec $\mathrm{rms}$ ) which allowed the optical identification on POSS-I prints based on positional coincidence. The analysis of these maps has also led to the exclusion of 53 sources from the sample because they were either lobes or because some sources joined to form a single source.

These sources were reobserved at the same frequency with the VLA in A configuration allowing a better resolution, i.e. HPBW 1.4 arcsec to be compared with 14 arcsecs of $\mathrm{C}$ configuration. A-configuration VLA maps will be presented elsewhere (Vigotti et al. 1996, in preparation).

The final radio sample contains 1050 radiosources. Table 1 shows the sample definitions, the number of sources and the solid angle covered in each subsample. All the sky area limits are at 1950.0 epoch, except the declination limits for sample 4, which correspond to the whole B3 catalogue, and are referred to 1978.0 epoch. The solid angles are corrected for the incompleteness regions of the B3 survey.

\subsection{Identifications}

Out of the original 352 identified objects, described in Vigotti et al. (1989), 183 were quasars candidates, defining a quasar candidate on the basis of its star-like appearance, regardless of the color, to avoid color biases.

These were examined using the positional coincidence between the optical and radio positions and the structural informations coming both from the A-configuration and C-configuration VLA maps. In order to build a complete sample of quasar candidates, we have accepted also identifications which are formally at low probability. For the unresolved sources the radio-optical rms displacement is $\sim 0$.'75 and the $3 \sigma$ radius is $\sim 2 . .5$, but we decided to extend the search up to $6^{\prime \prime}$. Infact, examining the radiooptical displacement histogram (see Fig. 1) for the 57 unresolved (in C-configuration) spectroscopically confirmed quasars, we see that the $3 \sigma$ choice would have implied a loss of $\sim 7 \%$ of the unresolved sources $(4 / 57)$ with a dis- placement greater than 2.5 . A posteriori looking the Aconfiguration maps we understand why the distribution is not gaussian. In all the four cases the quasar was coincident with the faint component of a double radiosource of $4-5$ arcseconds diameter, and with a very high flux ratio between the two radio components.

Furthermore, we obtained CCD images with the 1.5 meters Loiano Telescope of Bologna Observatory or with 3.5 meters telescope at Calar Alto of all the identified objects with uncertain optical classification from plates in the original sample, in order to be sure not to have missed any star-like object.

From the 183 quasar candidates in Vigotti et al. (1989) we excluded: (a) five candidates with $m_{\mathrm{r}} \geq$ $20.0 m \quad(0019+391, \quad 0800+399, \quad 0800+472, \quad 0805+406$ and $1357+392)$. (b) three candidates which from the A-configuration VLA maps turned into empty fields $(0209+390,1012+389$ and $1317+389)$. (c) three candidates which revealed to be plate-flaws from CCD images $(1033+408,1258+395$ and $1301+393)$.

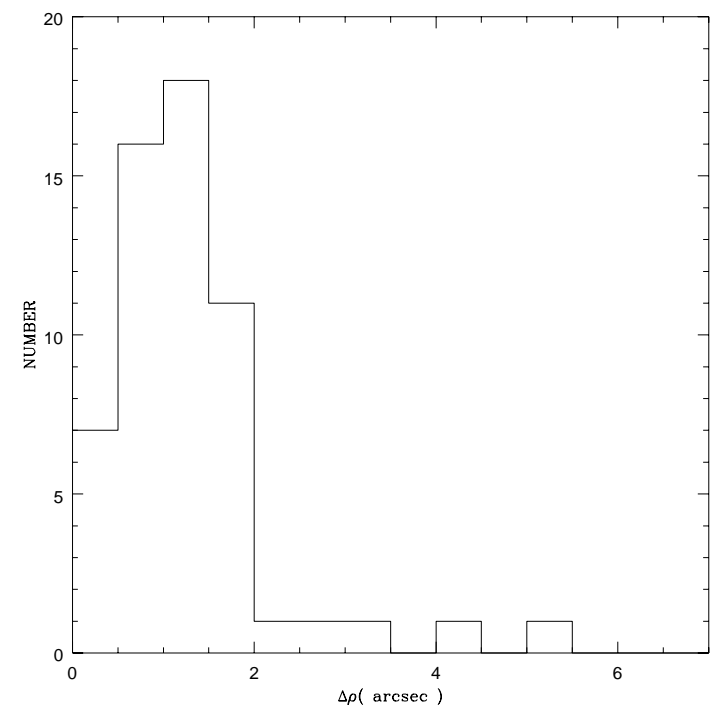

Fig. 1. Distribution of the radio-optical displacements for 57 unresolved quasars

\subsection{Magnitudes}

The candidates magnitudes in Vigotti et al. (1989) were estimates on POSS-I enlargements obtained by comparison with reference star images in the Selected Area 57. The accuracy of those estimated $R$ magnitudes is $0.5 \mathrm{~m}$. As machine measured $R$ magnitudes are available from the APM Catalogue (Irwin 1992) which have an rms error of $\sim 0.3 m$, we list these in Table 4 . There is a good agreement between the two magnitude scales (see Fig. 2) with a $0.024 m$ zero point. In two cases $(0143+446 \mathrm{~b}, 1342+389 \mathrm{a})$ we adopted the estimated magnitudes because APM was 
Table 1. Definition of the B3-VLA sample

\begin{tabular}{ccccc}
\hline Sample $\sharp$ & $\begin{array}{c}\text { Declination } \\
\text { range }\end{array}$ & $\begin{array}{c}\text { Flux }- \text { Density } \\
\text { range }(J y)\end{array}$ & $\begin{array}{c}\text { Number } \\
\text { of sources }\end{array}$ & $\begin{array}{c}\text { Area } \\
\text { Steradians }\end{array}$ \\
\hline 0 & $+39^{\circ} 38^{\prime}$ to $+40^{\circ} 00^{\prime}$ & $0.1 \leq S<0.2$ & 168 & 0.0125 \\
1 & $+38^{\circ} 50^{\prime}$ to $+40^{\circ} 00^{\prime}$ & $0.2 \leq S<0.4$ & 256 & 0.0405 \\
2 & $+38^{\circ} 00^{\prime}$ to $+40^{\circ} 48^{\prime}$ & $0.4 \leq S<0.8$ & 252 & 0.0971 \\
3 & $+38^{\circ} 00^{\prime}$ to $+44^{\circ} 30^{\prime}$ & $0.8 \leq S<1.6$ & 208 & 0.2341 \\
4 & $+37^{\circ} 15^{\prime}$ to $+47^{\circ} 37^{\prime}$ & $1.6 \leq S$ & 166 & 0.3813 \\
\hline
\end{tabular}

clearly wrong (they were giving the magnitude of the quasar plus a star few seconds apart). The sample is complete down to POSS-I limit in red, nominally 20.0; however a plate to plate variation of 0.25 magnitudes could affect the sample.

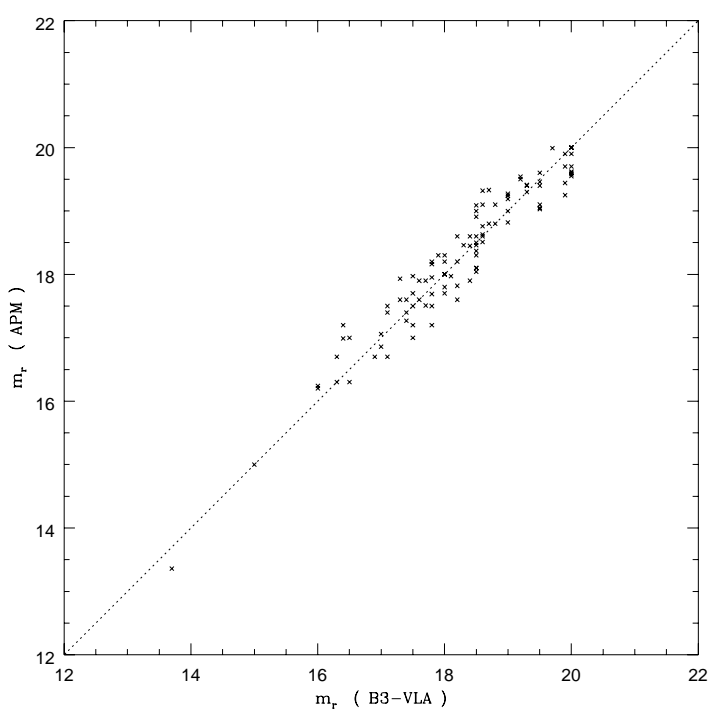

Fig. 2. B3 magnitudes versus APM magnitudes

\section{The sample completeness}

We can estimate the optical completeness of the sample, namely how many quasars are not included in our sample because their magnitude is fainter than 20.0 in red, as follows. In the sample with $S_{408} \geq 0.8 \mathrm{Jy}$ we can obtain a direct information: $55 \mathrm{EF}$ out of 210 have been observed spectroscopically and only $1 \mathrm{EF}$ turned out to be a quasar while all the other $\mathrm{EF}$ are high $z$ radiogalaxies (Djorgovski private communication 1996). We therefore estimate that about 4 quasars are fainter than the POSS-I limit. There will be 68 Quasars in this radio flux bin, regardless of the optical magnitude limit, 64 brigther than 20 magnitudes and 4 hidden among the Empty Fields. This is in good agreement with another direct information obtained by Grueff \& Vigotti (1975) by observing 113 Quasar candidates, at limiting flux of $0.9 \mathrm{Jy}$ at $408 \mathrm{MHz},(\sim 50 \%$ spectroscopically confirmed) down to $m_{v}=22.5$. They found only $4 \%$ of the quasar sample fainter than POSS-I limit. However the question of how many quasars are in the EF has different answers depending from the flux limit and from the frequency selection of the sample. The magnitude histograms in Fig. 3 show that for samples selected at low frequency the sample incompleteness is negligible at higher flux (3CR or B3-VLA at $S \geq 0.8 \mathrm{Jy}$ ). It is also evident that the median magnitude of the sample is becoming fainter in fainter flux bins. On the other hand lower flux samples $c$ and $d$, as defined in Fig. 3, show a clear cut in the right side of the histogram due to the POSS-I magnitude limit. An approximate evaluation of the number of the quasars in $\mathrm{EF}$ versus the sample flux can be obtained af follows. As hinted from Fig. 3 we can assume that the shape of the magnitudes distribution in different flux bins does not vary with flux but the whole histogram is shifted towards fainter magnitudes at lower fluxes. We compute $D_{\text {med }}$ : the difference of the median magnitude of $b$ and $c$ samples in Fig. 3, we shift the magnitude distribution of sample $b$ by $D_{\text {med }}$ toward fainter magnitudes and finally we assume that the fraction of quasars that are in EF in the sample $c$ is the same as for the new sample $b$. Than we recalculate the median of the sample $c$ and repeat the procedure. This allows to have a reasonable estimate of the number of quasars hidden in $\mathrm{EF}$ for different flux intervals (see Table 2).

\subsection{The final sample}

The final sample now contains 172 quasar candidates brighter than $m_{\mathrm{r}} \leq 20.0$. The quasar candidates sample is further subdivided into 120 blue starlike objects $(B)$ and 52 neutral color or red starlike objects $(N)$.

For all the candidates (except 35 with literature redshifts available) spectra were obtained at the $2.2 \mathrm{~m}$ and $3.5 \mathrm{~m}$ telescopes of Calar Alto during various observing runs. Some earlier data from these observations are described in Vigotti et al. (1990), and Lahulla et al. (1991). The remaining data are described in Sect. 3.

Table 3 presents the final assessment of the B3-VLA quasar candidates identifications after the identification revision and the spectroscopic observations.

The relatively high number of misidentifications with stars is due to acceptance of low-probability Identifications 
Table 2. Estimate of Quasars in EF

\begin{tabular}{lccccc}
\hline Flux Bin & Mean Flux (Jy) & $\begin{array}{c}\text { \# Quasars } \\
\text { with } m_{\mathrm{r}} \leq 20.0\end{array}$ & $\begin{array}{c}\text { Quasars } \\
\text { in EF }\end{array}$ & Median Mag. & $\begin{array}{c}\text { Quasar Percentage } \\
\text { in EF }\end{array}$ \\
\hline $0.1 \div 0.4$ & 0.22 & 30 & 13 & 19.6 & 30 \\
$0.4 \div 0.8$ & 0.53 & 31 & 12 & 19.3 & 28 \\
$\geq 0.8$ & 2.25 & 64 & 4 & 18.2 & 6 \\
\hline
\end{tabular}

Table 3. Identifications of the B3-VLA Quasar candidates sample

\begin{tabular}{ccccccc}
\hline COLOR & CANDIDATES & QUASARS & GALAXIES & BL-LACs & STARS & FEATURELESS \\
$B$ & 120 & 110 & 0 & 2 & 5 & 3 \\
$N$ & 52 & 15 & 22 & 1 & 13 & 1 \\
TOTAL & 172 & 125 & 22 & 3 & 18 & 4 \\
\hline
\end{tabular}
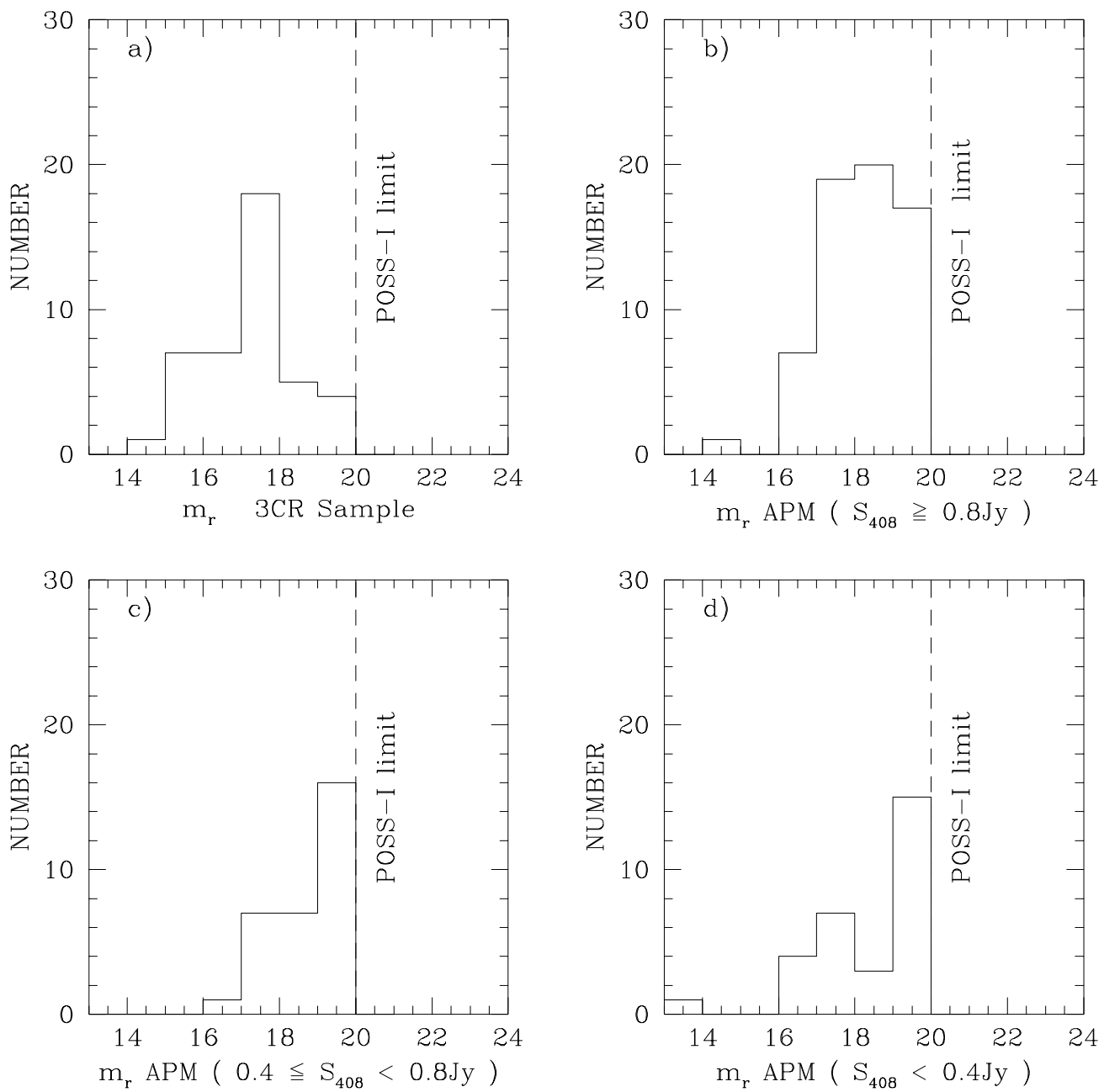

Fig. 3. The magnitudes distributions for different B3-VLA flux intervals and the 3 CR sample. a) $3 \mathrm{CR}$; b) $S_{408} \geq 0.8$ Jy; c) $0.4 \mathrm{Jy} \leq S_{408}<0.8 \mathrm{Jy} ;$ d) $0.1 \mathrm{Jy} \leq S_{408}<0.4 \mathrm{Jy}$ 
as described in Sect. 2.2. The 3 sources classified as BL Lac in Table 3 were previously known in literature. Four spectra are classified as featurless: two are possible BLLac candidates on the basis of their radio morphology: $2322+396$ and $2311+396 \mathrm{a}$. The former is unresolved (diam $\left.\leq 0.5^{\prime \prime}\right)$ and has flat radio spectral index $(0.0)$, while the latter is a less probable candidate, radio extended, diffuse and with a spectral index $=-0.9$. The remaining two objects with featureless spectra could be either galaxies or stars as they lack the typical radio characteristics of Bl-Lacs.

The final complete sample of quasars is presented in Table 4. Redshifts are available for 123 of the 125 spectroscopically confirmed quasar. The columns contain the following data: Column 1: name; Column 2: Right Ascention (1950.0) of the optical position (0.6 arcsec rms). Column 3: Declination (1950.0) of the optical position (0.6 arcsec rms). Column 4: Radio flux at $408 \mathrm{MHz}$. Column 5: APM red Magnitude. Column 6: Spectral Index. Column 7: Redshift. Column 8: Reference to published redshifts.

\section{Observations of the quasar candidates}

The observations of the quasar candidates candidates were accomplished with the Focal Reducer (Lenzen 1989) at the prime focus of the 3.5 meters telescope of the GermanSpanish Astronomical Center at Calar Alto (Spain) during six observing runs: October 1989, March 1990, October 1990, February 1991, September 1991 and November 1992.

Our spectra cover a wavelength range from about 4000 to $9000 \AA$ with resolution $\sim 12 \AA$ per pixel (except for the three last runs, when the focal reducer optics have been slightly modified giving a resolution of $\sim 16 \AA$ per pixel).

When the low dispersion spectrum showed no evident broad emission lines typical of quasars, the objects, with a few exceptions, were reobserved at higher dispersion (with a resolution of $\sim 4 \AA$ per pixel).

Flux calibration was obtained observing the following standard stars: Feige 98, Feige 15, Eggen 247 and HD 192281. Wavelength calibration spectra were obtained pointing to a screen in the dome illuminated by an $\mathrm{He}-\mathrm{Ar}$ lamp. We decided to take only one series of $\mathrm{He}-\mathrm{Ar}$ spectra at the end of each night, instead of one after each object, because of the conspicuous flexures arising from aiming at the screen near the dome floor. These flexures give rise to a wavelength shift which we corrected taking the NaI $5577 \AA$ sky line as a reference zero-point. Furthermore, at low resolution the paucity and the poor definition of some lines of the $\mathrm{He}-\mathrm{Ar}$ comparison spectrum near $4000 \AA$, has not allowed to obtain a good wavelength calibration in the blue region. In some cases the redshifts corresponding to the lines lying in the blue have been excluded in the calculation of the mean $z$.

Some representative spectra are shown in Fig. 4.

Table 5 lists the data for 77 of the 93 objects observed. The remaining 16 objects were spectroscopic stars and are listed in Table 6 together with other objects which changed the original identification, becoming "Empty Field" or "Galaxy".

The columns contain the following data: Column 1: name; Column 2: previous identification: $B$ blue object, $N$ red starlike object; Column 3: line identification; Column 4: observed wavelength; Column 5: rest wavelength adopted; Column 6: redshift computed for that line; Column 7: average redshift and eventually new spectroscopic identification, if not confirmed as Quasar.

To ease further studies of these objects, expecially of the few unsolved cases, we provide in Fig. 5 the finding charts for all the objects listed in the Table 5 and also for 8 objects listed in Table 6 identified as stars.

Table 6 lists objects whose identification changes due to spectroscopic and/or CCD observations. This table is intended to supplement table in Vigotti et al. (1989), where the optical coordinates are quoted.

\section{Comments on individual sources}

In this section some comments are collected for sources having peculiar or not well defined features in their spectra as well as details on a few objects whose identification changed from the original radio source identification.

0028+450: Our spectrum shows $\mathrm{MgI}(5175 \AA)$ and $\mathrm{NaI}$ (5892 $\AA$ ) at rest, thus we have classified this object as a star (see Table 6). However the identification is uncertain because Thomson et al. (1992) report the presence of one emission line which they identify as [OIII] (4983 $\AA$ at rest), thus classifying the object as a NELRG at $z=0.365$.

$0143+446 \mathrm{~b}$ : The original identification was 14 magnitude red stellar object. In a CCD we discovered a fainter object about 4 arcsec south which revealed to be the true identification, i.e. a quasar with $m_{\mathrm{r}} \sim 19$ (see Table 4 ).

0219+443: The spectrum only shows MgII, indicating a redshift of 0.852 , in good agreement with Djorgovski et al. (1990) who find 0.850 from a spectrum with five lines (see Table 5).

0226+467: Only one broad line at about $6200 \AA$ is present in our spectrum. We do not give the value of the redshift because of the poor Signal to Noise Ratio of the spectrum. If we identify the line as an MgII the redshift is $\sim 1.216$ (see Table 5).

0255+460: As mentioned earlier, our wavelength calibration has some uncertainties in the blue region of the spectrum. The feature at about $4240 \AA$ was identified as CIII, confirming the MgII at $6185 \AA$. The redshift reported in Table 5 is based on MgII only.

0724+396: The identification was with a galaxy of 16.5 mag which revealed to be a star. The Quasar is $5^{\prime \prime} \mathrm{SW}$ and closer to radio centroid (see Table 4).

0809+404: We report spectral data for this object, though it was not included in the 172 quasar candidates 

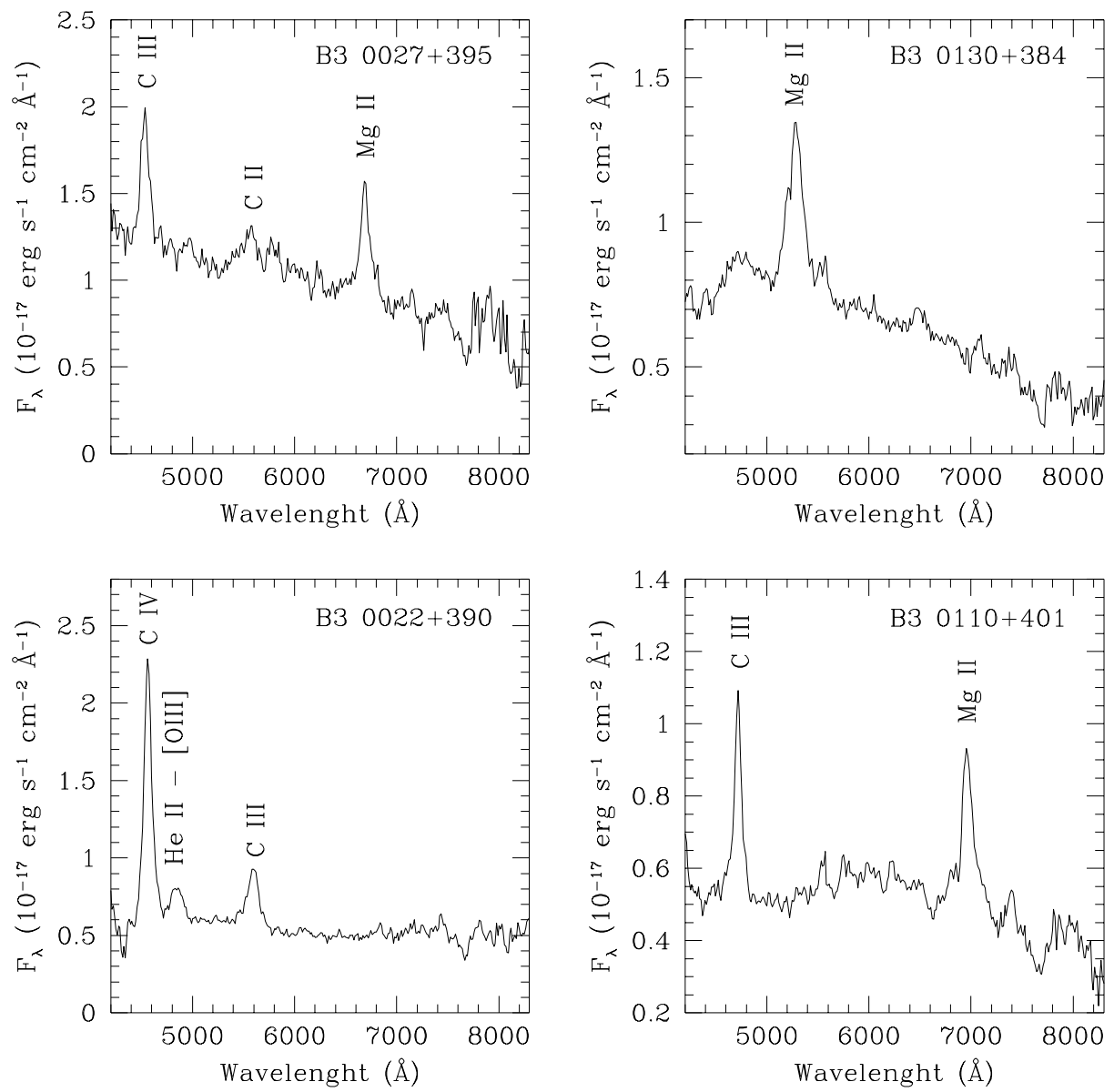

Fig. 4. Some representative spectra for the Quasars in the B3-VLA sample

sample. The original ID was G?, but it had stellar appearance on the CCD image. The spectrum classified it as Emission line Galaxy.

0912+392: The optical counterpart has been found $1.5 \mathrm{~m}$ fainter during the March 1990 observing run with respect to POSS-I.

0953+398: A broad line at about $4300 \AA$ has been identified as CIII, confirming that a line at $6099 \AA$ is MgII. The redshift derived from the CIII is not reported and the final value has been computed using MgII only (see Table 5).

1128+385: A line at about $4350 \AA$ has been identified as CIV, confirming the feature at $5221 \AA$ being CIII. The redshift relative to CIV is not reported because of problems with wavelength calibration at the blue end of the spectrum. The redshift is computed using CIII only (see Table 5).

1241+411: We report spectral data for this object, though it was not included in the 172 quasar candidates sample. The original ID was G?, but it had stellar appearance on the CCD image. The spectrum classified it as Emission line galaxy.
1416+400: A feature at about $4150 \AA$ has been identified with MgII but we didn't use it for the redshift calculation because of problems with wavelength calibration in that region of the spectrum. The other lines present in the spectrum give anyway a good $z$ measurement (see Table 5).

2316+398: The line at $7835 \AA$, identified as MgII, has a poor definition and it was not possible to obtain a secure value of the central wavelength, so we decided not to use it for the redshift determination. Thus the redshift has been computed using CIV only.

2322+396: The spectrum appears featureless but this may be due to the bad $\mathrm{S} / \mathrm{N}$ ratio (see Table 5 and Table 6 ), thus the identification is uncertain.

2329+398: The spectrum shows only one emission line at about $5010 \AA$ and wide enough to say that this is a Quasar. Since it was not possible to give a certain identification, the redshift is not reported.

\section{Discussion}

Out of 172 quasar candidates we have obtained a complete sample of 125 quasars. Figure 6 show the redshift 
distribution for the 123 quasars whose $z$ is known. The median redshift (dashed line) is 1.16. Of the remaining candidates 1 is a Seyfert galaxy, 5 are narrow emission line galaxies, 17 are normal galaxies, 17 have been reclassified as empty fields as the proposed identification turned out being a galactic star, 4 objects have featureless spectra so they can be Galaxies or Stars or Bl- Lacs.

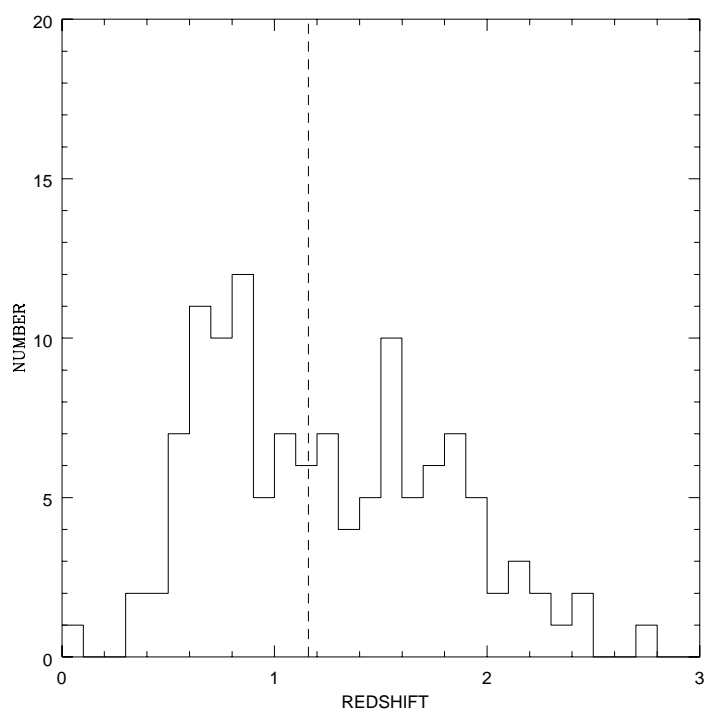

Fig. 6. Redshift distribution for the Quasars in the B3-VLA sample. The dashed vertical line indicates the median redshift: $z=1.16$

Acknowledgements. We would like to thank the GermanSpanish Astronomical Center, operated by the Max Plank Institute für Astronomie (Heidelberg), jointly with the Spanish National Commission for Astronomy.

\section{References}

Allington-Smith J.R., Spinrad H., Djorgowski S., Liebert J., 1988, MNRAS 234, 1091

Arp H., De Ruiter H.R., Willis A.G., 1979, A\&A 77, 86

Baldwin J.A, Burbidge E.M., Hazard C., Murdoch H.S., Robinson L.B., Wampler E.J., 1973, ApJ 185, 739

Barthel P.D., 1989, ApJ 336, 606

Blundell K.M., Rawlings S., Eales S.A., Lacy M., 1996, New results from complete samples of faint radio Galaxies and Quasars. In: Erkes R., Fanti C., Padrielli L. (eds.) Proc. IAU Symp. 175, Extra galactic radio sources. Kluwer, Dordrecht (in press)
Burbidge E.M., Kinman T.D., 1966, ApJ 145, 654

Burbidge E.M., 1968, ApJ 154, L109

Burbidge E.M., Strittmatter P.A., 1972, ApJ 174, L57

Djorgovski S., Thomson D.J., Vigotti M., Grueff G., 1990, PASP 102, 113

Djorgovski S., Thomson D., Maxfield L., Vigotti M., Grueff G., 1995, ApJS 101, 255

Ficarra A., Grueff G., Tomassetti G., 1985, A\&AS 59, 255

Grueff G., Vigotti M., 1975, A\&AS 20, 57

Hiltner W.A., Cowley A.P., Schild R.E., 1966, PASP 78, 464

Kapahi V.K., Athreya R.M., Subrahmanya C.R., et al., 1996, Radio structures of the MRC 1-Jy sources and the unification of radio Galaxies and Quasars. In: Erkes R., Fanti C., Padrielli L. (eds.), Proc. IAU Symp. 175, Extra galactic radio sources. Kluwer, Dordrecht (in press)

Kühr H., Schmidt G.D., 1990, AJ 99, 1

Irwin M., 1992, Gemini 37, 1 Newsletter of the Royal Greenwich Observatory

Lahulla J.F., Merighi R., Vettolani G., Vigotti M., 1991, A\&AS 88,525

Lenzen R., 1989, Focal Reducer for the prime focus of the $3.5 \mathrm{~m}$ Telescope, MPI fur Astronomie, Heidelberg

Lynds C.R., 1967, ApJ 147, 837

Owen F.N., Porcas R.W., Neff S.G., 1978, AJ 83, 1009

Schmidt M., 1965, ApJ 141, 1295

Schmidt M., 1974, ApJ 193, 505

Schmidt M., 1968, ApJ 151, 393

Singal A.K., 1993, MNRAS 263, 139

Smith H.E., Spinrad H., 1980, ApJ 236, 419

Steidel C.C., Sargent W.L.W., 1991, ApJ 382, 433

St:89 Stephens S.A., 1989, AJ 97, 10

Stickel M., Khur H., 1993, A\&AS 101, 521

Stickel M., Khur H., 1994, A\&AS 103, 349

Thomson D.J., Djorgovski S., Vigotti M., Grueff G., 1992, ApJS 81, 1

Tytler D., Fan X.M., 1992, ApJS 79, 1

Veron-Cetty M.P., Veron P., 1993, ESO scientific report 13

Vigotti M., Grueff G., Perley R., Clark B.G., Bridle A.H., 1989, AJ 98, 419

Vigotti M., Merighi R., Vettolani G., Lahulla J.F., LopezArroyo M., 1990, A\&AS 83, 205

Walsh D., Carswell R.F., 1982, MNRAS 200, 191

Walsh D., Beckers J.M., Carswell R.F., Weymann R.J., 1984, MNRAS 211, 105

Walsh D., Wills B.J., Wills D., 1979, MNRAS 189, 667

Webster R.L., Francis P.J., Peterson B.A., Drinkwater M.J., Masci F.J., 1995, Nat 375, 469

Wills B.J., Browne I.W.A., 1986, ApJ 302, 56

Wills D., Wills B.J., 1976, ApJS 31, 143

Wills D., Wills B.J., 1985, (private communication)

Xu W., Lawrence C.R., Readhead A.C.S., Pearson T.J., 1994, AJ 108, 395 
Table 4. The B3-VLA Quasars complete sample

\begin{tabular}{|c|c|c|c|c|c|c|c|}
\hline B3 name & R.A.(B 1950.0) & Dec. & $S 408 \mathrm{MHz}(\mathrm{Jy})$ & Sp.Ind. & $m_{\mathrm{r}}$ & $z$ & ref. \\
\hline \multicolumn{8}{|c|}{ Sample $0: 0.1 \leq S<0.2 \mathrm{Jy}$} \\
\hline $0107+398$ & 010754.05 & +395122.2 & 0.11 & 0.90 & 18.8 & 0.823 & \\
\hline $0158+394$ & 015900.20 & +392855.0 & 0.12 & 0.18 & 18.4 & 0.780 & \\
\hline $0209+394$ & 020933.86 & +392816.3 & 0.13 & 0.50 & 19.1 & 0.927 & \\
\hline $0739+398$ & 073913.28 & +395142.4 & 0.17 & 0.22 & 19.4 & 2.186 & \\
\hline $0754+394$ & 075438.20 & +392834.0 & 0.13 & 1.03 & 13.4 & 0.096 & Stephens 89 \\
\hline $1019+397$ & 101940.57 & +394700.8 & 0.17 & 0.86 & 16.2 & 0.605 & Vigotti 90 \\
\hline $1251+398$ & 125149.36 & +394938.6 & 0.15 & 0.93 & 19.5 & 2.104 & Lahulla 91 \\
\hline $1259+395$ & 125941.76 & +393132.0 & 0.10 & 1.05 & 19.5 & 0.577 & \\
\hline $1338+394$ & 133857.59 & +392957.4 & 0.18 & 0.68 & 19.2 & 0.580 & Lahulla 91 \\
\hline $1356+397$ & 135608.84 & +394716.8 & 0.12 & 0.73 & 19.6 & 0.594 & \\
\hline $1419+399$ & 141923.92 & +395708.0 & 0.14 & 0.44 & 17.6 & 0.622 & Vigotti 90 \\
\hline $2329+398$ & 232946.68 & +395043.8 & 0.18 & 1.12 & 20.0 & $?$ & \\
\hline \multicolumn{8}{|c|}{ Sample $1: 0.2 \leq S<0.4 \mathrm{Jy}$} \\
\hline $0022+399$ & 002203.51 & +395936.4 & 0.20 & 0.58 & 20.0 & 1.983 & \\
\hline $0034+393$ & 003454.24 & +3921 42.4 & 0.20 & -.08 & 17.0 & 1.937 & Baldwin 73 \\
\hline $0207+395$ & $\begin{array}{lll}02 & 07 & 07.47\end{array}$ & +393551.6 & 0.36 & 0.96 & 18.0 & 0.818 & \\
\hline $0225+389$ & 022552.93 & +385422.5 & 0.22 & 0.70 & 16.4 & 0.336 & Djorgovski 95 \\
\hline $0239+397$ & 023944.63 & +394300.7 & 0.37 & 0.86 & 20.0 & 1.159 & \\
\hline $0724+396$ & 072445.10 & +393638.0 & 0.24 & 1.09 & 19.8 & 2.753 & \\
\hline $0729+391$ & 072957.02 & +39 1134.9 & 0.26 & 0.43 & 17.9 & 0.663 & Djorgovski 90 \\
\hline $0824+397$ & 082448.59 & +394538.5 & 0.27 & 0.95 & 17.5 & 0.760 & Vigotti 90 \\
\hline $0912+392$ & 091255.43 & +39 1250.9 & 0.21 & 1.08 & 20.0 & 1.978 & \\
\hline $0935+397$ & 093533.06 & +394752.5 & 0.32 & 0.63 & 19.1 & 2.493 & \\
\hline $1116+392$ & 111619.50 & +391517.5 & 0.36 & 0.91 & 17.7 & 0.733 & Vigotti 90 \\
\hline $1123+395$ & 112345.85 & +393515.1 & 0.36 & 0.82 & 17.0 & 1.470 & Vigotti 90 \\
\hline $1204+399$ & 120404.55 & +395744.8 & 0.34 & 0.39 & 17.6 & 1.530 & Wills 85 \\
\hline $1212+389$ & 121243.28 & +385602.8 & 0.30 & 1.01 & 20.0 & 2.356 & \\
\hline $1300+397$ & 130029.06 & +394607.6 & 0.27 & 0.89 & 18.2 & 2.436 & Vigotti 90 \\
\hline $1349+388$ & 134910.63 & +385303.4 & 0.22 & -.05 & 20.0 & 1.888 & \\
\hline $2316+398$ & 231657.66 & +395338.5 & 0.29 & 0.93 & 20.0 & 1.846 & \\
\hline \multicolumn{8}{|c|}{ Sample $2: 0.4 \leq S<0.8 \mathrm{Jy}$} \\
\hline $0013+387$ & 001322.79 & +384347.0 & 0.74 & 0.98 & 18.5 & 1.721 & \\
\hline $0027+380$ & 002742.10 & +380047.2 & 0.58 & 1.08 & 19.3 & 1.066 & \\
\hline $0027+395$ & 002703.23 & +393203.4 & 0.41 & 0.69 & 18.5 & 1.388 & \\
\hline $0106+380$ & 010636.49 & +380047.8 & 0.45 & 0.74 & 16.9 & 0.583 & Wills 86 \\
\hline $0130+384$ & 013005.06 & +382538.1 & 0.53 & 1.01 & 19.3 & 0.889 & \\
\hline $0139+389 \mathrm{~A}$ & 013913.02 & +385734.4 & 0.43 & 0.67 & 20.0 & 1.106 & \\
\hline $0756+406$ & 075652.69 & +403810.6 & 0.50 & 0.86 & 18.8 & 2.016 & \\
\hline $0802+398$ & 080236.62 & +394922.4 & 0.41 & 1.01 & 19.6 & 1.800 & \\
\hline $0904+386$ & 090435.15 & +383946.5 & 0.43 & 0.83 & 17.7 & 1.730 & Wills 85 \\
\hline $0907+381$ & 090744.92 & +381132.5 & 0.43 & 0.39 & 17.3 & 2.160 & Wills 85 \\
\hline $0910+392$ & 091042.31 & +39 1437.7 & 0.41 & 1.08 & 19.2 & 0.638 & Lahulla 91 \\
\hline $0922+407$ & 092250.59 & +404248.6 & 0.48 & 0.33 & 19.6 & 1.876 & \\
\hline $0926+388$ & 092634.45 & +384913.6 & 0.50 & 1.09 & 18.3 & 1.630 & Lahulla 91 \\
\hline $0953+398$ & 095305.90 & +394932.1 & 0.50 & 0.41 & 19.7 & 1.179 & \\
\hline $1015+383$ & 101528.92 & +382034.5 & 0.64 & 0.86 & 17.7 & 0.380 & Wills 85 \\
\hline $1128+385$ & 112812.55 & +383152.3 & 0.52 & -.33 & 18.6 & 1.735 & Xu 94 \\
\hline
\end{tabular}


Table 4. continued

\begin{tabular}{|c|c|c|c|c|c|c|c|}
\hline B3 name & R.A. (B 1950.0) & Dec. & $S 408 \mathrm{MHz}(\mathrm{Jy})$ & Sp.Ind. & $m_{\mathrm{r}}$ & $z$ & ref. \\
\hline $1141+400$ & 114155.26 & +400002.2 & 0.41 & 0.95 & 18.5 & 0.907 & \\
\hline $1142+392$ & 114256.29 & +391326.0 & 0.57 & 1.02 & 18.0 & 2.276 & \\
\hline $1203+384$ & 120344.74 & +38 2917.3 & 0.67 & 0.98 & 18.0 & 0.838 & Vigotti 90 \\
\hline $1228+397$ & 122825.90 & +394633.8 & 0.60 & 0.89 & 17.9 & 2.217 & Vigotti 90 \\
\hline $1240+381$ & 124027.03 & +380725.1 & 0.41 & -.02 & 19.1 & 1.316 & \\
\hline $1312+393$ & 131249.52 & +39 1931.7 & 0.71 & 0.95 & 20.0 & 1.570 & \\
\hline $1317+380$ & 131736.20 & +380308.4 & 0.75 & 0.86 & 19.1 & 0.835 & Lahulla 91 \\
\hline $1348+392$ & 134823.61 & +39 1412.6 & 0.50 & 0.92 & 19.3 & 1.580 & Lahulla 91 \\
\hline $1355+380$ & 135530.54 & +380404.9 & 0.60 & 0.89 & 19.3 & 1.561 & \\
\hline $1357+394 \mathrm{~B}$ & 135756.50 & +392525.7 & 0.41 & 1.08 & 19.6 & 0.804 & \\
\hline $1416+400$ & 141656.66 & +400024.8 & 0.67 & 0.96 & 20.0 & 0.473 & \\
\hline $1417+385$ & 141743.05 & +383532.6 & 0.47 & -.37 & 19.4 & 1.832 & Lahulla 91 \\
\hline $1435+383$ & 143533.32 & +382043.7 & 0.54 & 0.85 & 17.1 & 1.600 & Vigotti 90 \\
\hline $2327+407$ & 232742.88 & +404751.8 & 0.48 & 0.56 & 18.0 & 1.220 & Vigotti 90 \\
\hline $2335+392$ & 233543.32 & +391655.5 & 0.56 & 0.93 & 18.8 & 1.059 & \\
\hline $2338+390$ & 233844.84 & +390149.3 & 0.62 & 0.98 & 20.0 & 0.816 & \\
\hline \multicolumn{8}{|c|}{ Sample $3: 0.8 \leq S<1.6 \mathrm{Jy}$} \\
\hline $0006+397$ & 000628.54 & +394505.2 & 1.15 & 0.66 & 19.5 & 1.830 & Wills 85 \\
\hline $0022+390$ & 002246.64 & +390258.9 & 1.10 & 0.10 & 18.6 & 1.932 & \\
\hline $0032+423$ & 003223.26 & +422149.6 & 0.92 & 0.94 & 18.2 & 1.588 & Burbidge 68 \\
\hline $0110+401$ & 011026.48 & +401019.6 & 1.08 & 0.65 & 19.7 & 1.479 & \\
\hline $0137+401$ & 013736.18 & +400903.9 & 0.82 & 0.85 & 18.9 & 1.62 & \\
\hline $0144+430$ & 014455.86 & +430448.1 & 0.92 & 1.01 & 19.3 & 1.790 & \\
\hline $0217+417$ & 021706.31 & +414356.9 & 0.85 & 0.94 & 18.4 & 1.430 & Arp 79 \\
\hline $0219+443$ & 021906.20 & +441917.8 & 0.89 & 1.17 & 17.9 & 0.852 & Djorgovski 90 \\
\hline $0224+393$ & 022400.97 & +3918 14.2 & 0.89 & 0.68 & 19.0 & 1.571 & \\
\hline $0249+383$ & 024958.98 & +382310.9 & 0.95 & 0.34 & 18.5 & 1.12 & \\
\hline $0701+392$ & 070105.07 & +391554.3 & 1.17 & 0.75 & 18.8 & 1.283 & Lahulla 91 \\
\hline $0726+431$ & 072616.70 & +430735.5 & 1.29 & 1.17 & 18.2 & 1.072 & Wills 76 \\
\hline $0739+397 B$ & 073945.93 & +394839.8 & 1.02 & 0.57 & 18.0 & 1.700 & Lahulla 91 \\
\hline $0829+425$ & 082926.28 & +423512.5 & 0.82 & 0.56 & 18.6 & 1.056 & \\
\hline $0836+426$ & 083635.50 & +423834.1 & 1.12 & 0.65 & 19.6 & 0.595 & \\
\hline $0849+424$ & 084915.71 & +422647.5 & 1.31 & 0.90 & 18.0 & 0.978 & Vigotti 90 \\
\hline $0922+422$ & 092247.56 & +421637.2 & 0.97 & 1.20 & 18.3 & 1.750 & Vigotti 90 \\
\hline $0922+425$ & 092212.43 & +423028.3 & 1.15 & 1.22 & 19.4 & 1.879 & \\
\hline $0951+408$ & 095139.03 & +405056.5 & 0.92 & 1.03 & 18.5 & 0.783 & \\
\hline $0955+387$ & 095501.70 & +384419.3 & 1.42 & 1.10 & 20.0 & 1.405 & Allington 88 \\
\hline $1030+415$ & 103007.78 & +413134.0 & 1.02 & 0.43 & 17.4 & 1.120 & Walsh 79 \\
\hline $1144+402$ & 114420.98 & +401513.9 & 0.93 & -.06 & 18.0 & 1.010 & Stickel 94 \\
\hline $1229+405$ & 122913.84 & +403402.4 & 0.91 & 0.98 & 19.0 & 0.649 & Lahulla 91 \\
\hline $1239+442 B$ & 123957.00 & +441234.3 & 1.21 & 0.92 & 17.8 & 0.610 & Vigotti 90 \\
\hline $1256+392$ & 125641.87 & +391622.9 & 0.80 & 1.03 & 17.9 & 0.978 & Vigotti 90 \\
\hline $1315+396$ & 131502.86 & +394115.4 & 1.16 & 0.49 & 17.6 & 1.560 & Vigotti 90 \\
\hline $1341+392$ & 134110.09 & +39 1335.3 & 1.22 & 1.01 & 19.9 & 0.768 & \\
\hline $1342+389 \mathrm{~A}$ & 134215.11 & +385631.3 & 0.86 & 1.09 & 17.6 & 1.533 & Lahulla 91 \\
\hline $1343+386$ & 134326.76 & +383812.1 & 1.52 & 0.48 & 17.5 & 1.844 & Schmidt 74 \\
\hline $1444+417 \mathrm{~A}$ & 144432.36 & +414550.1 & 1.58 & 1.03 & 17.8 & 0.675 & Lahulla 91 \\
\hline $2344+429$ & 234452.88 & +425412.7 & 0.92 & 0.58 & 18.3 & 1.556 & \\
\hline $2349+410$ & 234921.46 & +410434.0 & 1.38 & 0.98 & 19.8 & 2.046 & \\
\hline
\end{tabular}


Table 4. continued

\begin{tabular}{|c|c|c|c|c|c|c|c|}
\hline B3 name & R.A. (B 1950.0) & Dec. & $S 408 \mathrm{MHz}(\mathrm{Jy})$ & Sp.Ind. & $m_{\mathrm{r}}$ & $z$ & ref. \\
\hline \multicolumn{8}{|c|}{ Sample $4: S \geq 1.6 \mathrm{Jy}$} \\
\hline $0019+431$ & 001908.17 & +431147.1 & 2.22 & 1.34 & 19.4 & 1.050 & \\
\hline $0143+446 B$ & 014345.30 & +444021.0 & 1.71 & 0.99 & 17.8 & 0.813 & Thomson 92 \\
\hline $0157+442$ & 015732.74 & +441244.6 & 3.26 & 0.91 & 19.2 & 0.721 & \\
\hline $0226+467$ & 022605.20 & +464654.0 & 2.45 & 0.93 & 20.0 & $?$ & \\
\hline $0232+411 B$ & 023245.82 & +411013.5 & 2.75 & 0.99 & 17.4 & 0.500 & \\
\hline $0255+460$ & 025508.10 & +460406.0 & 1.74 & 0.77 & 20.0 & 1.21 & \\
\hline $0704+384$ & 070408.41 & +382656.8 & 2.87 & 1.06 & 17.4 & 0.579 & Schmidt 74 \\
\hline $0740+380 \mathrm{C}$ & 074056.75 & +380031.6 & 5.55 & 1.29 & 16.7 & 1.063 & Hiltner 66 \\
\hline $0821+394$ & 082137.31 & +392627.7 & 2.59 & 0.58 & 17.5 & 1.216 & Schmidt 74 \\
\hline $0821+447$ & 082150.27 & +444614.2 & 2.26 & 0.90 & 17.0 & 0.893 & Walsh 82 \\
\hline $0827+378$ & 082755.10 & +375218.2 & 5.17 & 0.80 & 18.2 & 0.914 & Lynds 67 \\
\hline $0859+470$ & 085939.90 & +470257.0 & 2.81 & 0.21 & 18.6 & 1.462 & Walsh 82 \\
\hline $0906+430$ & $0906 \quad 17.26$ & +430558.0 & 11.90 & 0.87 & 18.0 & 0.670 & Smith 80 \\
\hline $0913+391$ & 091339.50 & +390701.7 & 1.65 & 0.25 & 18.1 & 1.250 & Stickel 94 \\
\hline $0918+381$ & 091839.27 & +380657.7 & 2.45 & 1.07 & 19.1 & 1.108 & Lahulla 91 \\
\hline $0923+392$ & 092355.29 & +391523.4 & 3.33 & 0.09 & 16.9 & 0.698 & Burbidge 66 \\
\hline $0937+391$ & 093759.10 & +390731.0 & 1.88 & 0.86 & 17.5 & 0.618 & Burbidge 72 \\
\hline $0945+408$ & 094550.16 & +405344.1 & 2.45 & 0.51 & 17.2 & 1.252 & Walsh 82 \\
\hline $1007+417$ & 100726.13 & +414725.3 & 4.10 & 0.79 & 15.0 & 0.613 & Owen 78 \\
\hline $1020+400$ & 102014.56 & +400326.8 & 1.77 & 0.37 & 16.7 & 1.250 & Xu 94 \\
\hline $1105+392$ & 110551.57 & +391456.2 & 2.31 & 0.87 & 18.1 & 0.781 & Lahulla 91 \\
\hline $1109+437$ & 110952.28 & +434206.1 & 4.77 & 1.09 & 18.6 & 1.680 & Walsh 84 \\
\hline $1111+408$ & 111153.28 & +405341.9 & 10.92 & 1.16 & 16.3 & 0.734 & Schmidt 65 \\
\hline $1148+387$ & 114853.32 & +384234.4 & 1.83 & 0.94 & 16.2 & 1.303 & Steidel 91 \\
\hline $1148+477$ & 114832.28 & +474536.1 & 2.29 & 1.19 & 17.2 & 0.867 & Walsh 84 \\
\hline $1206+439 B$ & 120642.06 & +435601.9 & 5.69 & 0.85 & 16.7 & 1.400 & Schmidt 68 \\
\hline $1242+410$ & 124226.37 & +410430.2 & 2.01 & 0.25 & 19.1 & 0.811 & \\
\hline $1247+450 \mathrm{~A}$ & 124703.61 & +450110.9 & 1.68 & 0.73 & 17.2 & 0.799 & Walsh 84 \\
\hline $1258+404$ & 125813.99 & +402515.9 & 4.51 & 1.04 & 19.0 & 1.656 & Tytler 92 \\
\hline $1339+472$ & 133941.72 & +471224.6 & 2.21 & 1.02 & 19.9 & 0.502 & \\
\hline $2311+469$ & 231129.14 & +465554.5 & 4.34 & 0.69 & 18.1 & 0.745 & \\
\hline $2351+456$ & 235150.04 & +453623.0 & 2.21 & 0.27 & 20.0 & 2.000 & Stickel 93 \\
\hline
\end{tabular}


Table 5. Lines identification and redshifts

\begin{tabular}{|c|c|c|c|c|c|c|}
\hline B3 name & ID & Line & obs. & rest & $z$ & average $z$, new ID \\
\hline \multirow[t]{6}{*}{$0003+380$} & $\mathrm{~N}$ & [ O II ] & 4581 & 3727 & 0.229 & \\
\hline & & $\mathrm{H} \epsilon$ & 4752 & 3835 & 0.239 & Seyfert \\
\hline & & $\mathrm{H} \gamma$ & 5349 & 4340 & 0.232 & $\langle z\rangle=0.234 \pm 0.005$ \\
\hline & & $\mathrm{H} \beta$ & 5976 & 4861 & 0.229 & \\
\hline & & [ O III ] & 6155 & 5007 & 0.235 & \\
\hline & & $\mathrm{H} \alpha$ & 8140 & 6562 & 0.241 & \\
\hline \multirow[t]{3}{*}{$0013+387$} & $\mathrm{~B}$ & C IV & 4215 & 1549 & 1.721 & \\
\hline & & C III & 5181 & 1909 & 1.714 & $<z>=1.721 \pm 0.007$ \\
\hline & & C II & 6345 & 2326 & 1.728 & \\
\hline \multirow[t]{2}{*}{$0019+431$} & $\mathrm{~B}$ & $\mathrm{Mg}$ II & 5742 & 2798 & 1.052 & \\
\hline & & [ O III ] & 6419 & 3133 & 1.049 & $<z>=1.05 \pm 0.002$ \\
\hline $0020+437$ & $\mathrm{~B}$ & Featureless & & & & Gal/Star \\
\hline \multirow[t]{4}{*}{$0022+390$} & $\mathrm{~B}$ & C IV & 4540 & 1549 & 1.931 & \\
\hline & & HeII - [ OIII ] & & & & \\
\hline & & blended & 4847 & 1651 & 1.936 & $\langle z\rangle=1.932 \pm 0.003$ \\
\hline & & C III & 5593 & 1909 & 1.930 & \\
\hline \multirow[t]{2}{*}{$0022+399$} & $\mathrm{~B}$ & C III & 4621 & 1909 & 1.983 & \\
\hline & & C IV & 5696 & 1549 & 1.984 & $\langle z\rangle=1.983 \pm 0.001$ \\
\hline \multirow[t]{2}{*}{$0022+424$} & $\mathrm{~N}$ & Mg I (abs.) & 6215 & 5167 & 0.203 & Galaxy \\
\hline & & $\mathrm{Na}$ (abs.) & 7095 & 5892 & 0.204 & $\langle z\rangle=0.203 \pm 0.001$ \\
\hline \multirow{4}{*}{$0023+382$} & $\mathrm{~N}$ & $\mathrm{H} \theta$ (abs.) & 5118 & 3798 & 0.347 & \\
\hline & & $\mathrm{H} \delta$ (abs.) & 5577 & 4101 & 0.359 & Galaxy \\
\hline & & G band (abs.) & 5849 & 4304 & 0.359 & $\langle z>=0.355 \pm 0.006$ \\
\hline & & Mg I (abs.) & 6998 & 5167 & 0.354 & \\
\hline \multirow[t]{2}{*}{$0027+380$} & $\mathrm{~B}$ & $\mathrm{Mg}$ II & 5753 & 2798 & 1.056 & \\
\hline & & {$[\mathrm{O}$ II $]$} & 7738 & 3727 & 1.076 & $\langle z\rangle=1.066 \pm 0.014$ \\
\hline \multirow[t]{3}{*}{$0027+395$} & $\mathrm{~B}$ & C III & 4537 & 1909 & 1.377 & \\
\hline & & C II & 5575 & 2326 & 1.397 & $\langle z\rangle=1.388 \pm 0.011$ \\
\hline & & $\mathrm{Mg}$ II & 6688 & 2798 & 1.383 & \\
\hline $0035+385 a$ & $\mathrm{~B}$ & Featureless & & & & Gal/Star \\
\hline $0045+395$ & $\mathrm{~N}$ & Featureless & & & & BL-Lac \\
\hline \multirow[t]{2}{*}{$0107+398$} & $\mathrm{~B}$ & $\mathrm{Mg}$ II & 5087 & 2798 & 0.818 & \\
\hline & & {$[\mathrm{Ne} V]$} & 6260 & 3426 & 0.827 & $\langle z>=0.823 \pm 0.006$ \\
\hline \multirow[t]{2}{*}{$0110+401$} & $\mathrm{~B}$ & C III & 4719 & 1909 & 1.472 & \\
\hline & & $\mathrm{Mg}$ II & 6958 & 2798 & 1.487 & $\langle z\rangle=1.479 \pm 0.011$ \\
\hline \multirow[t]{2}{*}{$0130+384$} & $\mathrm{~B}$ & $\mathrm{Mg}$ II & 5280 & 2798 & 0.887 & \\
\hline & & {$[\mathrm{NeV}]$} & 6479 & 3426 & 0.891 & $\langle z\rangle=0.889 \pm 0.003$ \\
\hline \multirow[t]{2}{*}{$0137+401$} & $\mathrm{~B}$ & C III & 4982 & 1909 & 1.61 & \\
\hline & & $\mathrm{Mg}$ II & 7360 & 2798 & 1.63 & $\langle z\rangle=1.62 \pm 0.01$ \\
\hline \multirow[t]{2}{*}{$0139+389 a$} & $\mathrm{~B}$ & $\mathrm{Mg}$ II & 5895 & 2798 & 1.106 & \\
\hline & & Ne IV & 5105 & 2424 & 1.107 & $\langle z\rangle=1.106 \pm 0.001$ \\
\hline \multirow[t]{2}{*}{$0144+430$} & $\mathrm{~B}$ & $\mathrm{C}$ IV & 4327 & 1549 & 1.793 & \\
\hline & & C III & 5320 & 1909 & 1.787 & $\langle z\rangle=1.79 \pm 0.004$ \\
\hline \multirow[t]{3}{*}{$0153+417$} & $\mathrm{~N}$ & {$[\mathrm{Ne} V]$} & 5090 & 3426 & 0.485 & Em.lin.Gal. \\
\hline & & $\mathrm{O}$ II & 5535 & 3727 & 0.485 & $\langle z\rangle=0.488 \pm 0.005$ \\
\hline & & [ O III ] & 7477 & 5007 & 0.493 & \\
\hline \multirow[t]{8}{*}{$0157+442$} & $\mathrm{~N}$ & $\mathrm{Mg}$ II & 4800 & 2798 & 0.716 & \\
\hline & & {$[\mathrm{O}$ II } & 6397 & 3727 & 0.703 & \\
\hline & & {$[\mathrm{Ne}$ III $]$} & 6645 & 3870 & 0.717 & \\
\hline & & $\mathrm{H} \epsilon-\mathrm{Ne}$ III & & & & \\
\hline & & blended & 6830 & 3968 & 0.721 & $<z>=0.721 \pm 0.008$ \\
\hline & & $\mathrm{H} \delta$ & 7054 & 4101 & 0.720 & \\
\hline & & $\mathrm{H} \gamma$ & 7487 & 4340 & 0.725 & \\
\hline & & [ O III ] & 8643 & 5007 & 0.726 & \\
\hline
\end{tabular}


Table 5. continued

\begin{tabular}{|c|c|c|c|c|c|c|}
\hline B3 name & ID & Line & obs. & rest & $z$ & average $z$, new ID \\
\hline $0158+394$ & $\mathrm{~B}$ & $\mathrm{Mg}$ II & 4977 & 2798 & 0.78 & \\
\hline $0207+395$ & $\mathrm{~B}$ & $\mathrm{Mg}$ II & 5088 & 2798 & 0.818 & \\
\hline $0209+394$ & $\mathrm{~B}$ & $\mathrm{Mg}$ II & 5392 & 2798 & 0.927 & \\
\hline $0219+443$ & $\mathrm{~B}$ & $\mathrm{Mg}$ II & 5183 & 2798 & 0.852 & \\
\hline \multirow[t]{2}{*}{$0224+393$} & $\mathrm{~B}$ & C III & 4904 & 1909 & 1.569 & \\
\hline & & $\mathrm{Mg}$ II & 7200 & 2798 & 1.573 & $\langle z\rangle=1.571 \pm 0.003$ \\
\hline \multirow[t]{2}{*}{$0225+389$} & $\mathrm{~B}$ & [ O III ] & & & & \\
\hline & & blend & 6700 & 4983 & 0.344 & \\
\hline $0226+467$ & $\mathrm{~N}$ & $?$ & $\approx 6200$ & & & Quasar \\
\hline \multirow{9}{*}{$0232+411 b$} & $\mathrm{~B}$ & $\mathrm{Mg}$ II & 4195 & 2798 & 0.499 & \\
\hline & & {$[\mathrm{Ne} \mathrm{V}]$} & 5145 & 3426 & 0.502 & \\
\hline & & {$[\mathrm{O}$ II $]$} & 5598 & 3727 & 0.502 & \\
\hline & & [ $\mathrm{Ne}$ III ] & 5804 & 3870 & 0.500 & \\
\hline & & $\mathrm{H} \epsilon-\mathrm{Ne}$ III & & & & $\langle z\rangle=0.500 \pm 0.002$ \\
\hline & & blended & 5942 & 3968 & 0.497 & \\
\hline & & $\mathrm{H} \delta$ & 6151 & 4101 & 0.500 & \\
\hline & & $\mathrm{H} \gamma$ & 6516 & 4340 & 0.501 & \\
\hline & & [ O III ] & 6546 & 4363 & 0.500 & \\
\hline $0239+397$ & $\mathrm{~B}$ & $\mathrm{Mg}$ II & 6040 & 2798 & 1.159 & \\
\hline $0249+383$ & $\mathrm{~B}$ & $\mathrm{Mg}$ II & 5930 & 2798 & 1.12 & \\
\hline \multirow[t]{2}{*}{$0255+460$} & $\mathrm{~B}$ & C III & $\approx 4240$ & 1909 & & \\
\hline & & $\mathrm{Mg}$ II & 6185 & 2798 & 1.21 & $z=1.21$ \\
\hline $0709+409$ & $\mathrm{~N}$ & & & & & Galaxy from CCD image \\
\hline \multirow[t]{4}{*}{$0724+396$} & $\mathrm{~N}$ & $\begin{array}{l}\text { Ly } \alpha \\
\text { O IV - Si IV }\end{array}$ & 4575 & 1216 & 2.762 & \\
\hline & & blended & 5280 & 1405 & 2.758 & $<z>=2.753 \pm 0.013$ \\
\hline & & C IV & 5790 & 1549 & 2.738 & \\
\hline & & C III & 7120 & 1909 & 2.730 & \\
\hline \multirow[t]{4}{*}{$0739+398$} & $\mathrm{~B}$ & O IV - Si IV & & & & \\
\hline & & blended & 4476 & 1405 & 2.186 & \\
\hline & & C IV & 4932 & 1549 & 2.184 & $\langle z\rangle=2.186 \pm 0.002$ \\
\hline & & C III & 6088 & 1909 & 2.189 & \\
\hline \multirow[t]{2}{*}{$0756+406$} & $\mathrm{~B}$ & C IV & 4716 & 1549 & 2.044 & \\
\hline & & C III & 5724 & 1909 & 1.988 & $\langle z\rangle=2.016 \pm 0.039$ \\
\hline \multirow[t]{4}{*}{$0802+398$} & $\mathrm{~B}$ & C IV & 4339 & 1549 & 1.801 & \\
\hline & & He II & 4593 & 1640 & 1.801 & $<z>=1.800 \pm 0.002$ \\
\hline & & C III & 5339 & 1909 & 1.797 & \\
\hline & & $\mathrm{Mg}$ II & 7862 & 2798 & 1.801 & \\
\hline \multirow[t]{7}{*}{$0809+404$} & $\mathrm{G}$ & {$[\mathrm{Ne} \mathrm{V}]$} & 5585 & 3426 & 0.630 & \\
\hline & & {$[\mathrm{O}$ II $]$} & 5781 & 3727 & 0.551 & \\
\hline & & [ Ne III ] & 6000 & 3870 & 0.550 & Seyfert \\
\hline & & $\mathrm{H} \delta$ & 6333 & 4101 & 0.544 & $\langle z\rangle=0.551 \pm 0.003$ \\
\hline & & $\mathrm{H} \beta$ & 7552 & 4861 & 0.554 & \\
\hline & & [ O III ] & 7697 & 4959 & 0.552 & \\
\hline & & [ O III ] & 7773 & 5007 & 0.552 & \\
\hline $0820+431$ & $\mathrm{~N}$ & & & & & Galaxy from CCD image \\
\hline \multirow[t]{2}{*}{$0829+425$} & $\mathrm{~B}$ & $\mathrm{Mg}$ II & 5750 & 2798 & 1.055 & \\
\hline & & [ O III ] & 6443 & 3133 & 1.056 & $\langle z\rangle=1.056 \pm 0.001$ \\
\hline \multirow[t]{3}{*}{$0836+426$} & $\mathrm{~B}$ & $\mathrm{Mg}$ II & 4466 & 2798 & 0.596 & \\
\hline & & [O II & 5929 & 3727 & 0.591 & $\langle z\rangle=0.595 \pm 0.004$ \\
\hline & & [ O III ] & 6974 & 4363 & 0.598 & \\
\hline $0841+403$ & $\mathrm{~N}$ & & & & & Galaxy from CCD image \\
\hline $0902+414$ & $\mathrm{~N}$ & & & & & Galaxy from CCD image \\
\hline $0904+399$ & $\mathrm{~N}$ & & & & & Galaxy from CCD image \\
\hline
\end{tabular}


Table 5. continued

\begin{tabular}{|c|c|c|c|c|c|c|}
\hline B3 name & ID & Line & obs. & rest & $z$ & average $z$, new ID \\
\hline \multirow[t]{3}{*}{$0912+392$} & $\mathrm{~B}$ & Ly $\alpha$ & 3639 & 1216 & 1.993 & \\
\hline & & C IV & 4624 & 1549 & 1.985 & $\langle z\rangle=1.978 \pm 0.019$ \\
\hline & & C III & 5643 & 1909 & 1.956 & \\
\hline \multirow[t]{4}{*}{$0922+407$} & $\mathrm{~B}$ & C IV & 4448 & 1549 & 1.872 & \\
\hline & & [ O III ] & 4754 & 1663 & 1.859 & $\langle z\rangle=1.876 \pm 0.01$ \\
\hline & & C III & 5478 & 1909 & 1.869 & \\
\hline & & $\mathrm{Mg}$ II & 8075 & 2798 & 1.886 & \\
\hline \multirow[t]{2}{*}{$0922+425$} & $\mathrm{~N}$ & C IV & 4450 & 1549 & 1.873 & \\
\hline & & C III & 5510 & 1909 & 1.886 & $\langle z\rangle=1.879 \pm 0.01$ \\
\hline \multirow[t]{5}{*}{$0935+397$} & $\mathrm{~N}$ & Ly $\alpha$ & 4249 & 1216 & 2.494 & \\
\hline & & O IV - Si IV & & & & \\
\hline & & blended & 4883 & 1405 & 2.488 & $\langle z\rangle=2.493 \pm 0.003$ \\
\hline & & C IV & 5413 & 1549 & 2.494 & \\
\hline & & C III & 6674 & 1909 & 2.496 & \\
\hline \multirow[t]{5}{*}{$0936+405$} & $\mathrm{~N}$ & {$[\mathrm{O}$ II $]$} & 4444 & 3727 & 0.192 & \\
\hline & & [ O III ] & 5899 & 5007 & 0.178 & Em.lin.Gal. \\
\hline & & $\mathrm{O} I]$ & 7485 & 6300 & 0.188 & $\langle z\rangle=0.186 \pm 0.005$ \\
\hline & & $\mathrm{H} \alpha$ & 7779 & 6562 & 0.185 & \\
\hline & & S II & 7981 & 6731 & 0.186 & \\
\hline \multirow[t]{2}{*}{$0951+408$} & $\mathrm{~B}$ & $\mathrm{Mg}$ II & 4989 & 2798 & 0.783 & \\
\hline & & {$[\mathrm{NeV}]$} & 6108 & 3426 & 0.783 & $<z>=0.783$ \\
\hline \multirow[t]{2}{*}{$0953+398$} & $\mathrm{~N}$ & C III & $\approx 4300$ & 1909 & & \\
\hline & & $\mathrm{Mg}$ II & 6099 & 2798 & 1.179 & \\
\hline \multirow[t]{2}{*}{$1128+385$} & $\mathrm{~B}$ & C IV & $\approx 4350$ & 1549 & & \\
\hline & & C III & 5221 & 1909 & 1.735 & \\
\hline \multirow[t]{3}{*}{$1128+455$} & $\mathrm{~N}$ & {$[\mathrm{O}$ II ] } & 5217 & 3727 & 0.409 & Em.lin.Gal. \\
\hline & & [ O III ] & & & & $\langle z\rangle=0.404 \pm 0.007$ \\
\hline & & blend & 7023 & 4983 & 0.400 & \\
\hline $1141+400$ & $\mathrm{~N}$ & $\mathrm{Mg}$ II & 5336 & 2798 & 0.907 & \\
\hline \multirow[t]{3}{*}{$1141+466$} & $\mathrm{G}$ & $\mathrm{H} \beta$ (abs.) & 5261 & 4861 & 0.092 & \\
\hline & & Mg I (abs.) & 5680 & 5167 & 0.098 & $\langle z\rangle=0.092 \pm 0.009$ \\
\hline & & $\mathrm{H} \alpha$ (abs.) & 7206 & 6562 & 0.098 & \\
\hline \multirow[t]{4}{*}{$1142+392$} & $\mathrm{~B}$ & O IV - Si IV & & & & \\
\hline & & blended & 4604 & 1405 & 2.277 & \\
\hline & & C IV & 5075 & 1549 & 2.276 & $\langle z\rangle=2.276 \pm 0.001$ \\
\hline & & C III & 6254 & 1909 & 2.276 & \\
\hline \multirow[t]{6}{*}{$1212+389$} & $\mathrm{~B}$ & O IV - Si IV & & & & \\
\hline & & blended & 4703 & 1405 & 2.347 & \\
\hline & & C IV & 5218 & 1549 & 2.369 & \\
\hline & & HeII - [ O III ] & & & & $\langle z\rangle=2.356 \pm 0.013$ \\
\hline & & blended & 5520 & 1651 & 2.342 & \\
\hline & & C III & 6427 & 1909 & 2.367 & \\
\hline \multirow[t]{4}{*}{$1241+411$} & $\mathrm{G}$ & {$[\mathrm{O}$ III $]$} & 3907 & 3133 & 0.247 & \\
\hline & & [ O III ] & & & & $\langle z\rangle=0.259 \pm 0.011$ \\
\hline & & blend & 6278 & 4983 & 0.260 & $\mathrm{AGN}$ \\
\hline & & $\mathrm{H} \alpha$ & 8333 & 6562 & 0.269 & \\
\hline \multirow[t]{2}{*}{$1242+410$} & $\mathrm{~B}$ & $\mathrm{Mg}$ II & 5052 & 2798 & 0.806 & \\
\hline & & [ O II ] & 6768 & 3727 & 0.816 & $\langle z\rangle=0.811 \pm 0.007$ \\
\hline $1259+395$ & $\mathrm{~B}$ & $\mathrm{Mg}$ II & 4412 & 2798 & 0.577 & \\
\hline \multirow[t]{2}{*}{$1312+393$} & $\mathrm{~B}$ & C III & 4923 & 1909 & 1.579 & \\
\hline & & $\mathrm{Mg}$ II & 7160 & 2798 & 1.559 & $<z>=1.57 \pm 0.01$ \\
\hline \multirow[t]{5}{*}{$1339+472$} & $\mathrm{~B}$ & $\mathrm{Mg}$ II & $\approx 4250$ & 2798 & & \\
\hline & & {$[\mathrm{Ne} \mathrm{V}]$} & 5128 & 3426 & 0.500 & \\
\hline & & {$[\mathrm{O}$ II $]$} & 5582 & 3727 & 0.501 & \\
\hline & & {$[\mathrm{Ne}$ III $]$} & 5797 & 3870 & 0.499 & \\
\hline & & $\mathrm{H} \epsilon-\mathrm{Ne}$ III & & & & $\langle z\rangle=0.502 \pm 0.003$ \\
\hline
\end{tabular}


Table 5. continued

\begin{tabular}{|c|c|c|c|c|c|c|}
\hline$\overline{\text { B3 name }}$ & ID & Line & obs. & rest & $z$ & average $z$, new ID \\
\hline & & blended & 5942 & 3968 & 0.497 & \\
\hline & & $\mathrm{H} \delta$ & 6156 & 4101 & 0.501 & \\
\hline & & $\mathrm{H} \gamma$ & 6522 & 4340 & 0.503 & \\
\hline & & $\mathrm{H} \beta$ & 7332 & 4861 & 0.508 & \\
\hline & & [ O III ] & 7536 & 5007 & 0.505 & \\
\hline \multirow[t]{4}{*}{$1341+392$} & $\mathrm{~N}$ & $\mathrm{Mg}$ II & 4945 & 2798 & 0.767 & \\
\hline & & {$[\mathrm{Ne} V]$} & 6044 & 3426 & 0.764 & \\
\hline & & {$[\mathrm{O}$ II $]$} & 6595 & 3727 & 0.769 & $\langle z\rangle=0.768 \pm 0.003$ \\
\hline & & {$[\mathrm{Ne}$ III $]$} & 6855 & 3870 & 0.772 & \\
\hline \multirow[t]{2}{*}{$1349+388$} & $\mathrm{~B}$ & C IV & 4475 & 1549 & 1.889 & \\
\hline & & C III & 5510 & 1909 & 1.886 & $\langle z\rangle=1.888 \pm 0.002$ \\
\hline \multirow[t]{3}{*}{$1355+380$} & $\mathrm{~B}$ & [ O III ] & 4246 & 1663 & 1.553 & \\
\hline & & C III & 4880 & 1909 & 1.556 & $\langle z\rangle=1.561 \pm 0.011$ \\
\hline & & $\mathrm{Mg}$ II & 7200 & 2798 & 1.574 & \\
\hline \multirow[t]{6}{*}{$1356+397$} & $\mathrm{~B}$ & $\mathrm{Mg}$ II & 4438 & 2798 & 0.586 & \\
\hline & & {$[\mathrm{Ne} V]$} & 5445 & 3426 & 0.589 & \\
\hline & & {$[\mathrm{O}$ II $]$} & 5945 & 3727 & 0.595 & $\langle z\rangle=0.594 \pm 0.006$ \\
\hline & & {$[\mathrm{Ne}$ III $]$} & 6177 & 3870 & 0.596 & \\
\hline & & $\mathrm{H} \beta$ & 7781 & 4861 & 0.601 & \\
\hline & & [ O III ] & 8005 & 5007 & 0.599 & \\
\hline \multirow[t]{3}{*}{$1357+394 b$} & $\mathrm{~B}$ & $\mathrm{Mg}$ II & 5032 & 2798 & 0.798 & \\
\hline & & {$[\mathrm{Ne} V]$} & 6188 & 3426 & 0.806 & $\langle z\rangle=0.804 \pm 0.005$ \\
\hline & & {$[\mathrm{O}$ II $]$} & 6733 & 3727 & 0.806 & \\
\hline \multirow[t]{10}{*}{$1416+400$} & $\mathrm{~B}$ & $\mathrm{Mg}$ II & $\approx 4150$ & 2798 & & \\
\hline & & {$[\mathrm{Ne} V]$} & 5045 & 3426 & 0.472 & \\
\hline & & {$[\mathrm{O}$ II $]$} & 5485 & 3727 & 0.472 & \\
\hline & & [ Ne III ] & 5701 & 3870 & 0.473 & \\
\hline & & $\mathrm{H} \epsilon-\mathrm{Ne}$ III & & & & \\
\hline & & blended & 5836 & 3968 & 0.471 & $\langle z\rangle=0.473 \pm 0.003$ \\
\hline & & $\mathrm{H} \delta$ & 6037 & 4101 & 0.473 & \\
\hline & & $\mathrm{H} \gamma$ & 6410 & 4340 & 0.470 & \\
\hline & & $\mathrm{H} \beta$ & 7194 & 4861 & 0.479 & \\
\hline & & [ O III ] & 7410 & 5007 & 0.479 & \\
\hline $1432+397 \mathrm{~b}$ & $\mathrm{~N}$ & & & & & Galaxy from CCD image \\
\hline $2311+396 a$ & $\mathrm{~N}$ & Featureless & & & & Gal/Star/BL-Lac \\
\hline \multirow{4}{*}{$2311+469$} & $\mathrm{~B}$ & $\mathrm{Mg}$ II & 4885 & 2798 & 0.746 & \\
\hline & & He II & 5596 & 3203 & 0.747 & \\
\hline & & {$[\mathrm{Ne} \mathrm{V}$} & 5973 & 3426 & 0.743 & $\langle z\rangle=0.745 \pm 0.002$ \\
\hline & & {$[\mathrm{Ne}$ III $]$} & 6744 & 3870 & 0.743 & \\
\hline \multirow[t]{2}{*}{$2316+398$} & $\mathrm{~B}$ & C IV & 4409 & 1549 & 1.846 & \\
\hline & & $\mathrm{Mg}$ II & 7835 & 2798 & & \\
\hline $2322+396$ & $\mathrm{~B}$ & Featureless & & & & BL-Lac ? \\
\hline $2329+398$ & $\mathrm{~B}$ & $?$ & 5010 & & & Quasar \\
\hline \multirow[t]{3}{*}{$2335+392$} & $\mathrm{~B}$ & $\mathrm{Mg}$ II & 5751 & 2798 & 1.055 & \\
\hline & & {$[\mathrm{O}$ III $]$} & 6452 & 3133 & 1.059 & $\langle z\rangle=1.059 \pm 0.003$ \\
\hline & & {$[\mathrm{Ne} \mathrm{V}]$} & 7062 & 3426 & 1.061 & \\
\hline $2338+390$ & $\mathrm{~B}$ & $\mathrm{Mg}$ II & 5980 & 2798 & 0.82 & \\
\hline \multirow[t]{2}{*}{$2344+429$} & $\mathrm{~B}$ & C III & 4866 & 1909 & 1.549 & \\
\hline & & $\mathrm{Mg}$ II & 7172 & 2798 & 1.563 & $<z>=1.556 \pm 0.01$ \\
\hline \multirow[t]{2}{*}{$2349+410$} & $\mathrm{~B}$ & C IV & 4710 & 1549 & 2.041 & \\
\hline & & C III & 5825 & 1909 & 2.051 & $\langle z\rangle=2.046 \pm 0.007$ \\
\hline
\end{tabular}


Table 6. Quasar candidates with different spectroscopic identification

\begin{tabular}{|c|c|c|c|c|}
\hline B3 name & Orig. ID & $m_{\mathrm{r}}$ & New ID & ref. \\
\hline $0000+394$ & $\mathrm{~N}$ & 17.5 & STAR & \\
\hline $0003+380$ & $\mathrm{~N}$ & 17.0 & SEYFERT & \\
\hline $0020+437$ & B & 19.5 & GAL/STAR & \\
\hline $0022+424$ & $\mathrm{~N}$ & 19.5 & GAL & \\
\hline $0023+382$ & $\mathrm{~N}$ & 19.0 & GAL & \\
\hline $0028+450$ & $\mathrm{~N}$ & 17.4 & STAR & \\
\hline $0035+385 \mathrm{~A}$ & B & 18.5 & GAL/STAR & \\
\hline $0045+395$ & $\mathrm{~N}$ & 16.0 & BL-Lac & Djorgovski 95 \\
\hline $0153+417$ & $\mathrm{~N}$ & 19.5 & GAL & \\
\hline $0209+390$ & B & 20.0 & $\mathrm{EF}$ & \\
\hline $0217+395$ & $\mathrm{~N}$ & 18.5 & STAR & \\
\hline $0218+402 \mathrm{~A}$ & $\mathrm{~N}$ & 13.5 & STAR & \\
\hline $0219+428 \mathrm{~A}$ & B & 16.0 & BL-Lac & Veron 93 \\
\hline $0228+392$ & $\mathrm{~N}$ & 13.5 & STAR & \\
\hline $0709+409$ & $\mathrm{~N}$ & 20.0 & GAL & \\
\hline $0739+397 \mathrm{~A}$ & $\mathrm{~N}$ & 15.7 & STAR & \\
\hline $0812+406$ & $\mathrm{~N}$ & 18.4 & STAR & \\
\hline $0814+425$ & B & 19.1 & BL-Lac & Kühr 90 \\
\hline $0820+431$ & $\mathrm{~N}$ & 19.5 & GAL & \\
\hline $0841+403$ & $\mathrm{~N}$ & 19.5 & GAL & \\
\hline $0902+414$ & $\mathrm{~N}$ & 17.5 & GAL & \\
\hline $0902+416$ & $\mathrm{~N}$ & 19.5 & STAR & \\
\hline $0904+399$ & $\mathrm{~N}$ & 17.5 & GAL & \\
\hline $0936+405$ & $\mathrm{~N}$ & 18.5 & GAL & \\
\hline $1012+389$ & $\mathrm{~N}$ & 20.0 & $\mathrm{EF}$ & \\
\hline $1033+408$ & $\mathrm{~N}$ & 20.0 & $\mathrm{EF}$ & \\
\hline $1128+455$ & $\mathrm{~N}$ & 18.5 & GAL & \\
\hline $1245+396$ & B & 17.7 & STAR & \\
\hline $1258+395$ & $\mathrm{~N}$ & 18.5 & $\mathrm{EF}$ & \\
\hline $1301+393$ & $\mathrm{~N}$ & 19.5 & $\mathrm{EF}$ & \\
\hline $1317+389$ & $\mathrm{~B}$ & 19.7 & $\mathrm{EF}$ & \\
\hline $1401+387$ & B & 15.8 & STAR & \\
\hline $1402+382$ & $\mathrm{~B}$ & 16.5 & STAR & \\
\hline $1432+397 B$ & $\mathrm{~N}$ & 18.5 & GAL & \\
\hline $1457+388 \mathrm{~A}$ & B & 18.9 & STAR & \\
\hline $2301+394 \mathrm{~B}$ & $\mathrm{~N}$ & 20.0 & STAR & \\
\hline $2301+398$ & $\mathrm{~N}$ & 19.5 & STAR & \\
\hline $2301+430$ & $\mathrm{~N}$ & 16.0 & STAR & \\
\hline $2311+396 \mathrm{~A}$ & $\mathrm{~N}$ & 18.5 & GAL/STAR/BL-Lac & \\
\hline $2322+396$ & $\mathrm{~B}$ & 17.8 & BL-Lac? & \\
\hline $2330+435$ & $\mathrm{~N}$ & 15.4 & STAR & \\
\hline $2336+381$ & $\mathrm{~N}$ & 17.5 & STAR & \\
\hline $2355+398$ & $\mathrm{~B}$ & 18.0 & STAR & \\
\hline
\end{tabular}



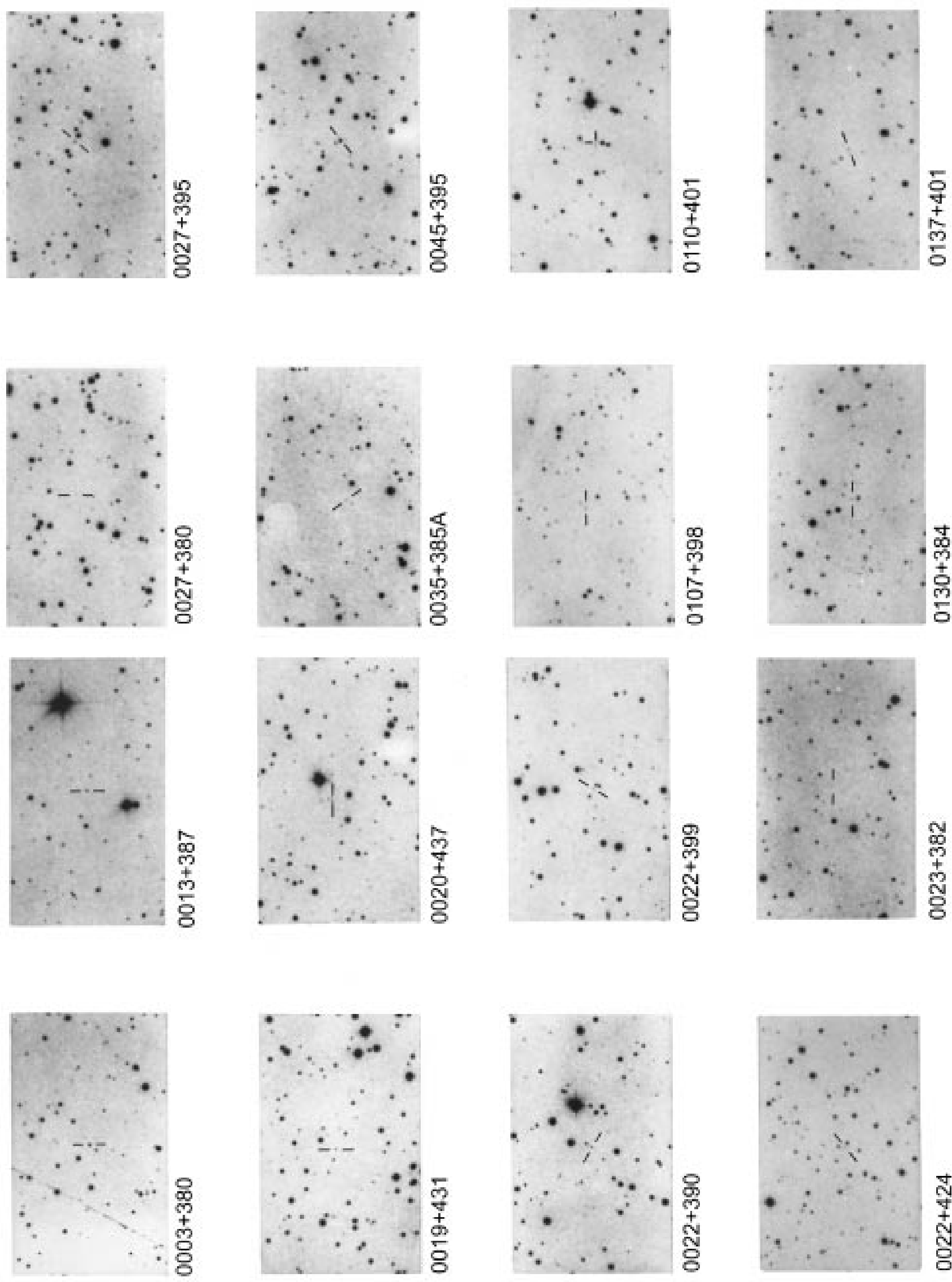

Fig. 5. Finding charts reproduced from the POSS-I for the objects listed in Table 5 

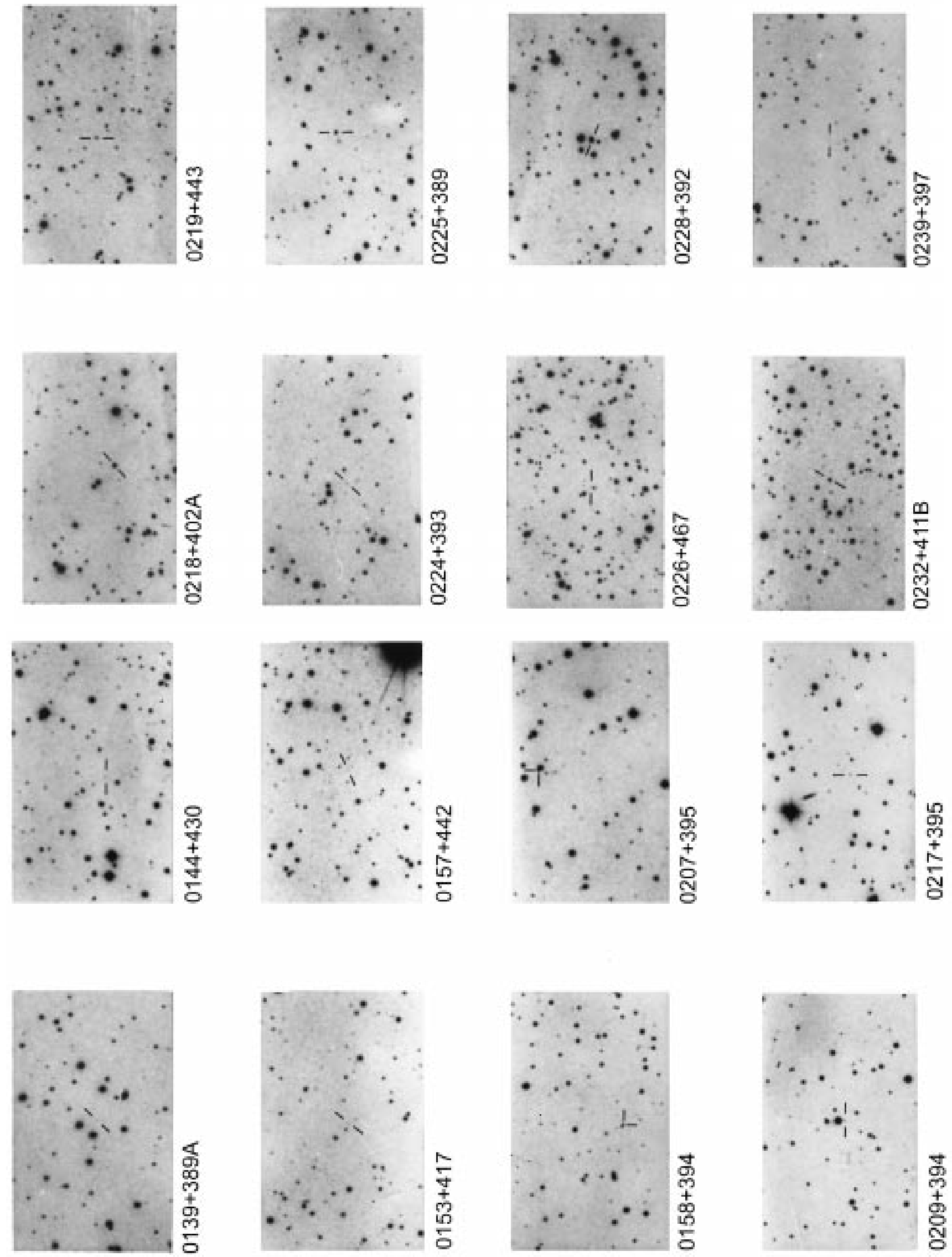

Fig. 5. continued 

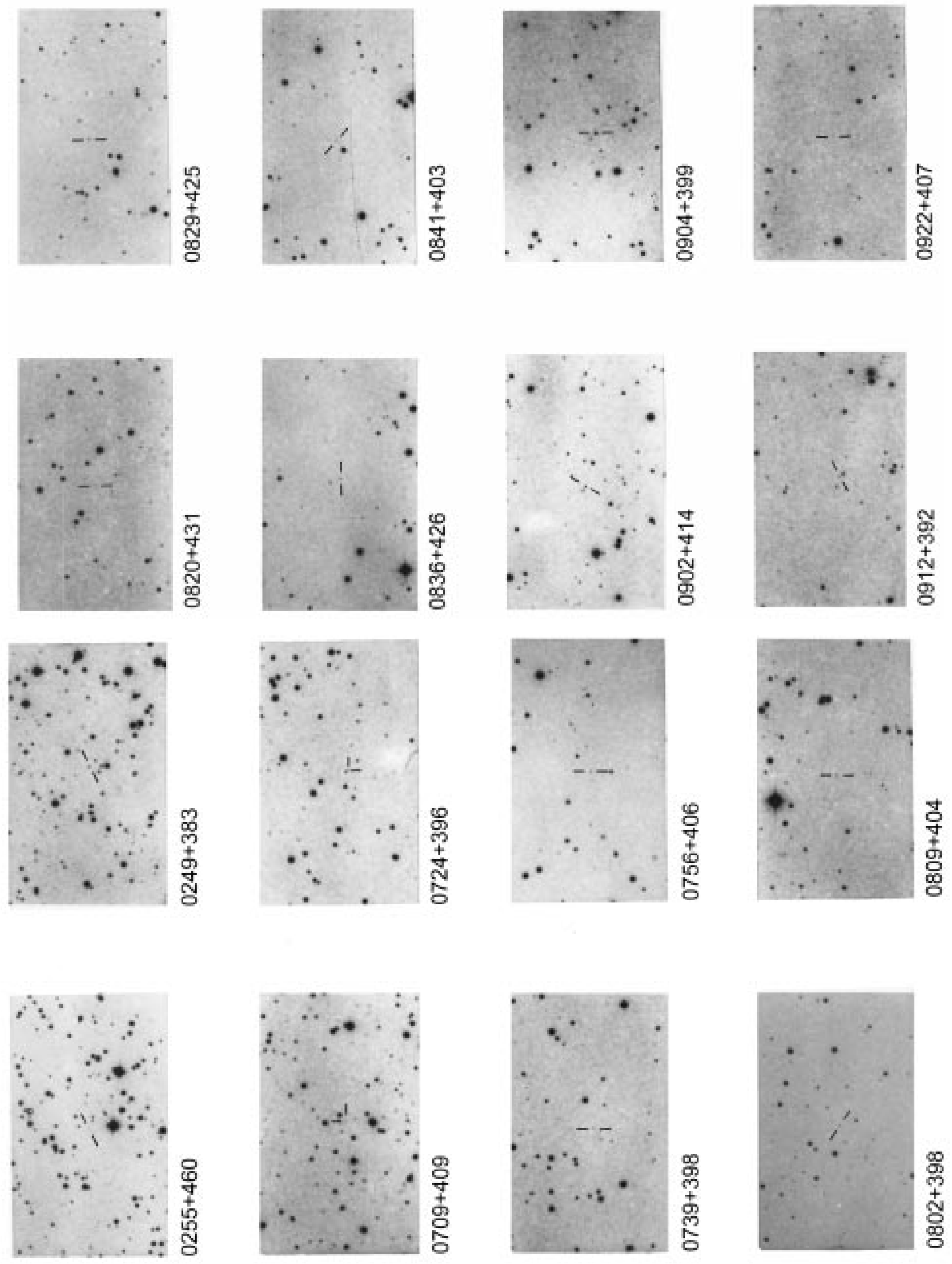

Fig. 5. continued 

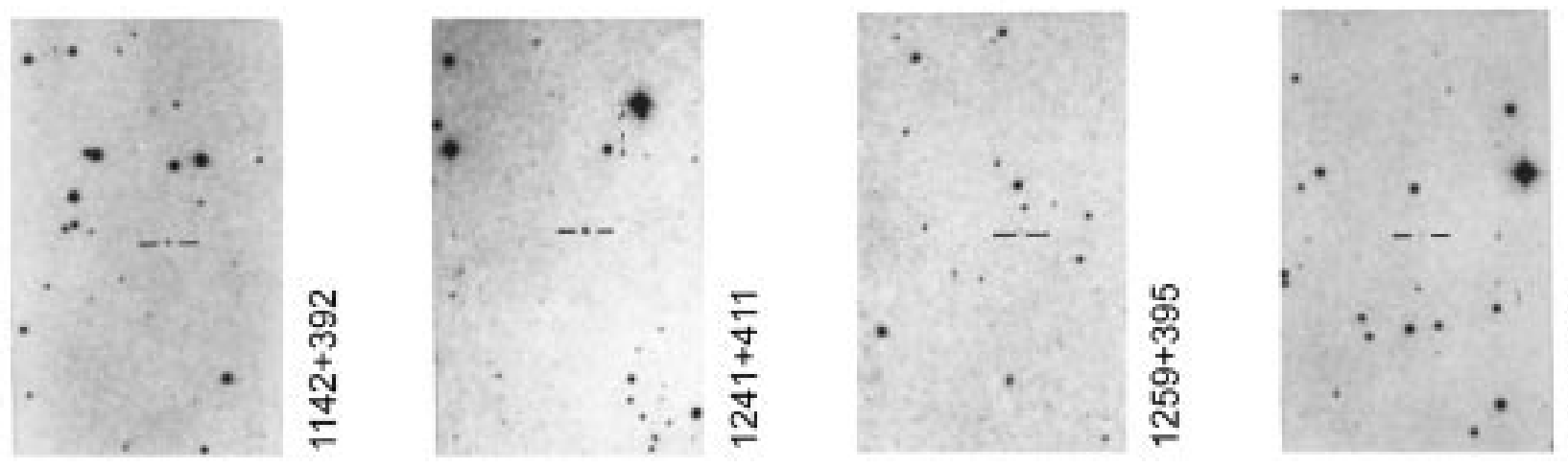

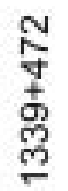
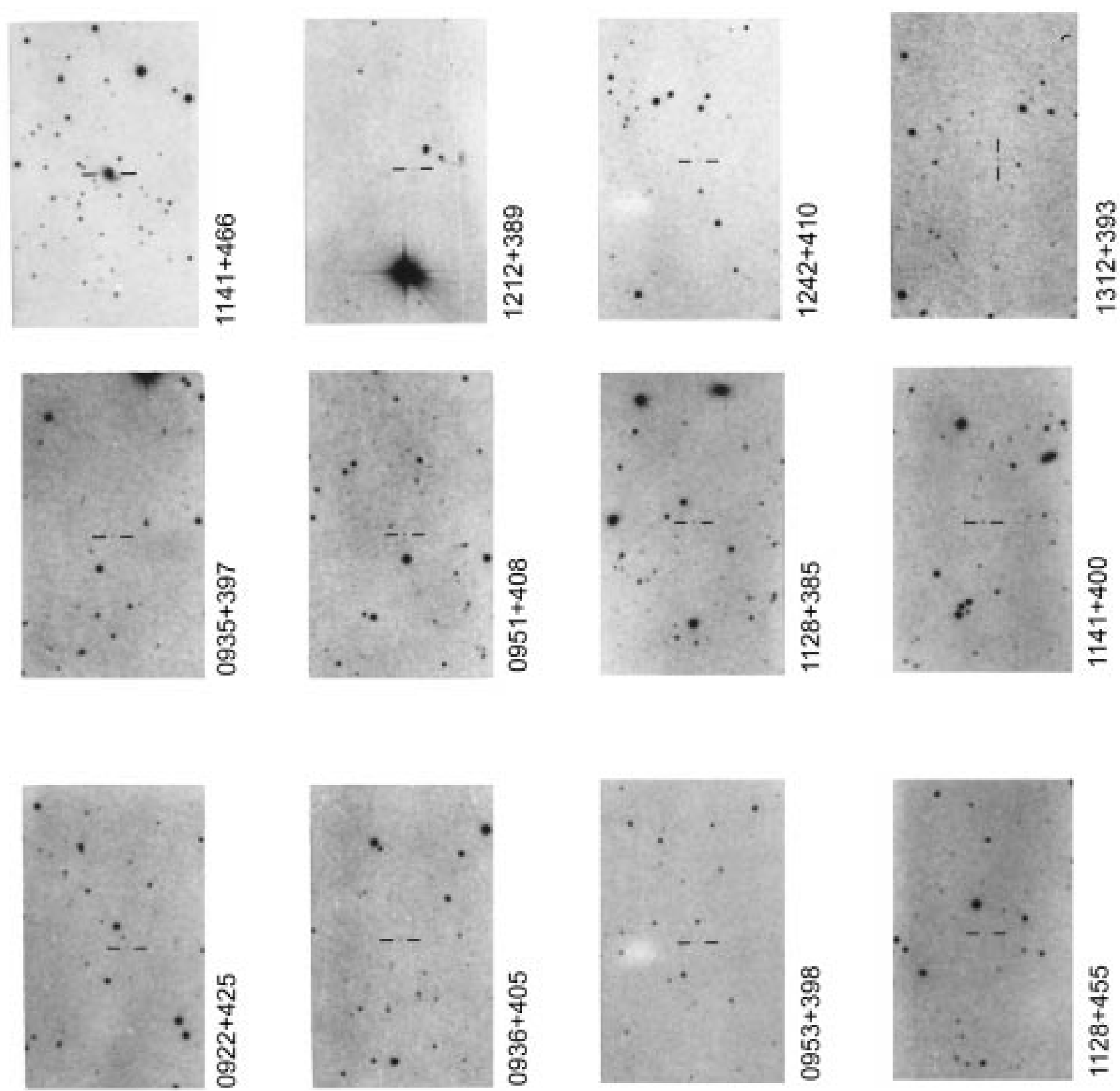

Fig. 5. continued 

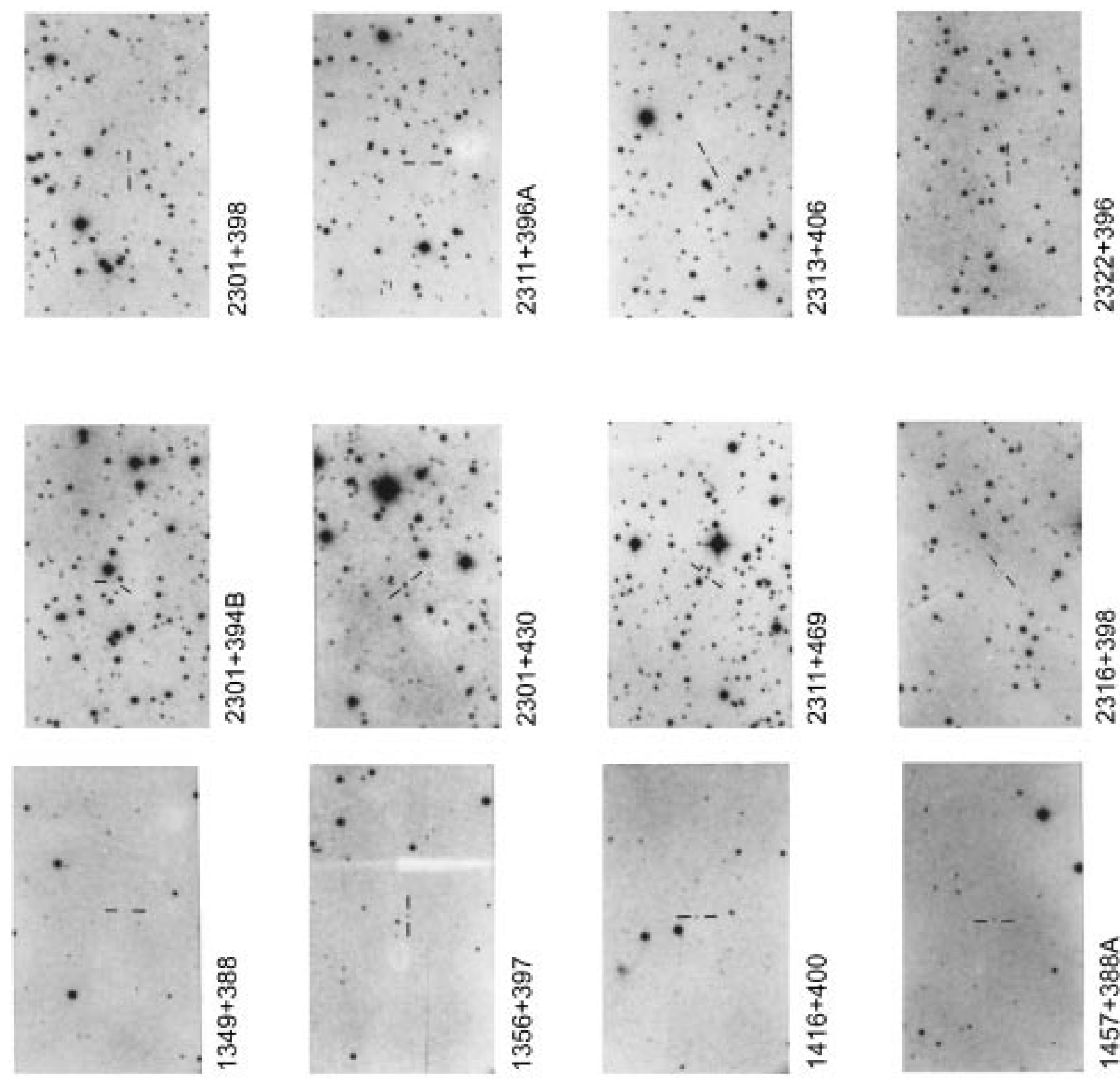

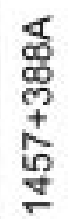
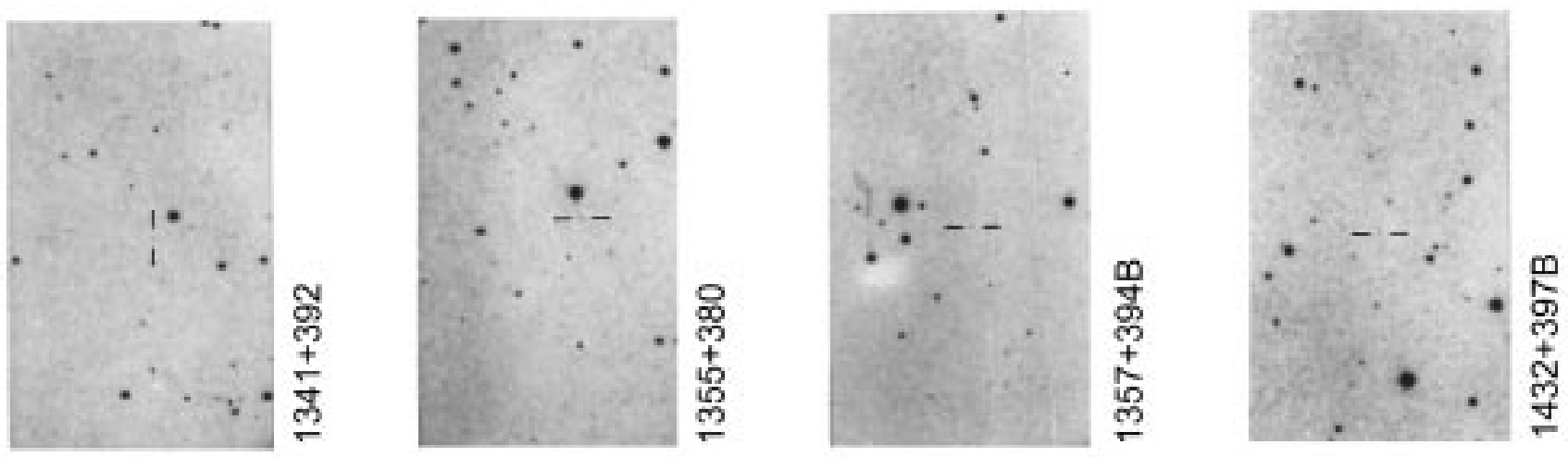

Fig. 5. continued 

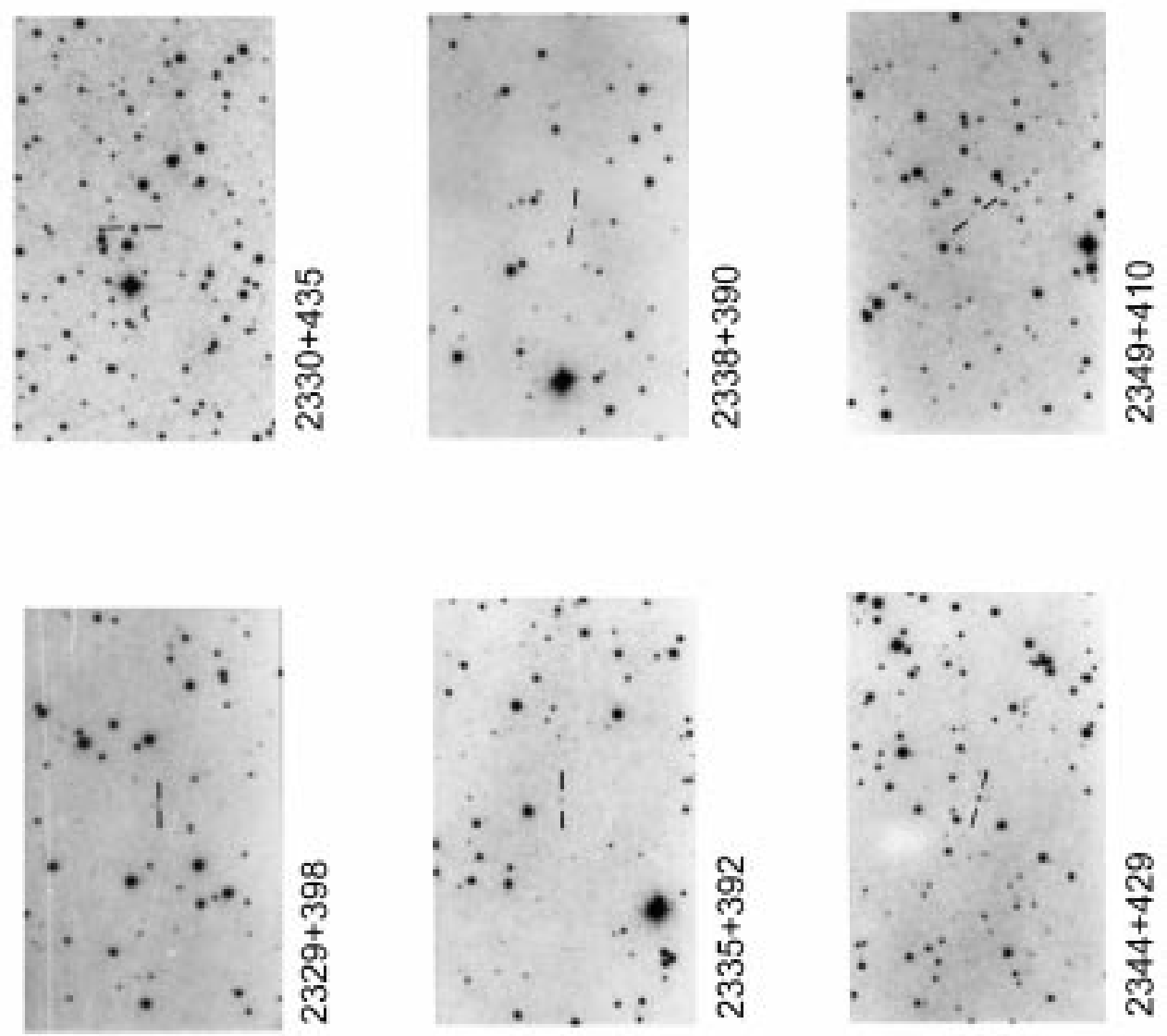

Fig. 5. continued 